\title{
The Autism Protein Ube3A/E6AP Remodels Neuronal Dendritic Arborization via Caspase-Dependent Microtubule Destabilization
}

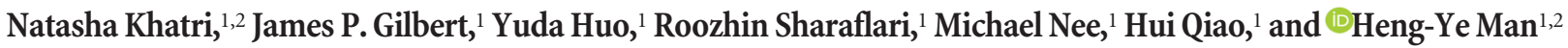 \\ ${ }^{1}$ Department of Biology, Boston University, Boston, Massachusetts 02215, and ${ }^{2}$ Department of Pharmacology \& Experimental Therapeutics, Boston \\ University School of Medicine, Boston, Massachusetts 02118
}

UBE3A gene copy number variation and the resulting overexpression of the protein E6AP is directly linked to autism spectrum disorders (ASDs). However, the underlying cellular and molecular neurobiology remains less clear. Here we report the role of ASD-related increased dosage of Ube3A/E6AP in dendritic arborization during brain development. We show that increased E6AP expression in primary cultured neurons leads to a reduction in dendritic branch number and length. The E6AP-dependent remodeling of dendritic arborization results from retraction of dendrites by thinning and fragmentation at the tips of dendrite branches, leading to shortening or removal of dendrites. This remodeling effect is mediated by the ubiquitination and degradation of XIAP (X-linked inhibitors of aptosis protein) by E6AP, which leads to activation of caspase-3 and cleavage of microtubules. In vivo, male and female Ube3A 2X ASD mice show decreased XIAP levels, increased caspase- 3 activation, and elevated levels of tubulin cleavage. Consistently, dendritic branching and spine density are reduced in cortical neurons of Ube3A 2X ASD mice. In revealing an important role for Ube3A/E6AP in ASD-related developmental alteration in dendritic arborization and synapse formation, our findings provide new insights into the pathogenesis of Ube3A/E6APdependent ASD.

Key words: ASD; dendritic remodeling; developmental disorder; neuronal development; UBE3A/E6AP; ubiquitination

Significance Statement

Copy number variation of the UBE3A gene and aberrant overexpression of the gene product E6AP protein is a common cause of autism spectrum disorders (ASDs). During brain development, dendritic growth and remodeling play crucial roles in neuronal connectivity and information integration. We found that in primary neurons and in Ube $3 \mathrm{~A}$ transgenic autism mouse brain, overexpression of E6AP leads to significant loss of dendritic arborization. This effect is mediated by the ubiquitination of XIAP (X-linked inhibitor of aptosis protein) by E6AP, subsequent activation of caspases, and the eventual cleavage of microtubules, leading to local degeneration and retraction at the tips of dendritic branches. These findings demonstrate dysregulation in neuronal structural stability as a major cellular neuropathology in ASD.

\section{Introduction}

Autism spectrum disorders (ASDs) are clinically characterized by decreased communication abilities, impaired social interaction,

Received May 29, 2017; revised Sept. 27, 2017; accepted 0ct. 31, 2017.

Author contributions: N.K. and H.-Y.M. designed research; N.K., J.P.G., Y.H., and H.Q. performed research; Y.H. and H.Q. contributed unpublished reagents/analytic tools; N.K., J.P.G., R.S., and M.N. analyzed data; N.K. and H.-Y.M. wrote the paper.

We thank Dr. Andrea LeBlanc (McGill University), for providing the cleaved tubulin antibody Tub $\Delta$ Casp6, and Dr. Wilson Wong (Boston University), for providing the pTRE-mCherry plasmids. We also thank Dr. Gregory Dillon and Dr. Angela Ho (Boston University) for technical assistance with live imaging. We thank members of the Man Laboratory for discussion and helpful comments. This work was supported by National Institutes of Health Grant R01 MH079407 (H.-Y.M.).

The authors declare no competing financial interests.

Correspondence should be addressed to Heng-Ye Man at the above address. E-mail: hman@bu.edu. and the occurrence of repetitive behaviors (Levy et al., 2009). ASD is becoming a common neurodevelopmental disorder with a prevalence of $\sim 1$ in 70 individuals in the United States (Zablotsky et al., 2015). ASDs are typically diagnosed during the first 3 years of life, a period of extensive neuronal development, including dendritic and synaptic growth and refinement (McGee et al., 2014). As a common comorbidity shared with other neurodevelopmental disorders, ASDs present a scientific and therapeutic challenge.

$U B E 3 A$ is a major ASD gene located on chromosome $15 \mathrm{q} 11$ 13 , a region that has been in focus of genetic studies on autism 
susceptibility. $U B E 3 A$ is genetically imprinted, where only the maternal copy of the gene is expressed in the brain and the paternal copy is silenced (Albrecht et al., 1997). Copy number variations of $U B E 3 A$ are strongly implicated in ASDs, whereas deletions of the gene are involved in Angelman syndrome (AS; Williams et al., 2006). Individuals with an additional maternal copy of $U B E 3 A$ (dup15) and those with two extra copies from an isodicentric extranumery chromosome (idic15) both display autism penetrance (Hogart et al., 2010). These studies suggest the importance of $U B E 3 A$ gene dosage in brain development. The protein product of UBE3A, E6AP, is a HECT (homologous to the E6-AP $\mathrm{C}$ terminus) family E3 ligase (Crinelli et al., 2008; Kim and Huibregtse, 2009). However, the neurobiological function of this E3 ligase in ASDs remains less clear.

As dendrites are the main structures that receive information in neurons, the complexity of dendrites and synaptic spine number determine neuronal connectivity and communication. Development, refinement, and maintenance of the neuronal dendritic arbors are therefore crucial to normal brain function. The formation of dendritic arborization is a dynamic process. Following early developmental growth, mature branching patterns are established not only by neurite elongation and new branch formation, but also by branch retraction and elimination, where dendritic branches are shortened or removed by dendrite remodeling to optimize the connectivity and function of neural circuits (Kozlowski and Schallert, 1998; Cline, 2001; Puram et al., 2010; Tao and Rolls, 2011). Dendritic remodeling and retraction is a general developmental process occurring in neurons from insects to mammals (Zehr et al., 2006; Parrish et al., 2007; Tao and Rolls, 2011). Selective and active elimination of dendrites after initial cortical-layer establishment is essential in shaping the functional architecture of the cortex (Koester and O'Leary, 1992). In the hippocampus and prefrontal cortex of ASD patients, significant changes in dendritic branching and spine density have been observed (Williams et al., 1980; Raymond et al., 1996; Mukaetova-Ladinska et al., 2004). Consistently, ASD genes, including FMRP and Mecp2, have been shown to affect neuronal morphology in animal models (Irwin et al., 2001; Berman et al., 2012; de Anda et al., 2012; Pathania et al., 2014). However, the role of E6AP in neuron morphogenesis and maturation in relation to ASD and the underlying cellular and molecular mechanisms remain largely unknown.

We therefore investigated the role of ASD-related increased dosage of E6AP in dendritic arborization and synapse maturation during brain development. Using primary neuron cultures, we found that elevated E6AP expression causes remodeling of dendritic arborization with a significant reduction in dendrite length and branch numbers, and with dendritic retraction occurring from the distal end via thinning and local degeneration. After exploring the signaling cascade, we found that E6AP targets XIAP (X-linked inhibitor of aptosis protein) for ubiquitination and degradation, and subsequently elevates levels of activated caspase-3, leading to microtubule cleavage and eventual retraction and removal of dendritic branches. In the Ube $3 A$ ASD mouse model, cortical neurons display a significant reduction in dendritic branches and spine density. Importantly, we found that the same signaling cascade involved in dendritic remodeling in vitro is used in the ASD mice. These novel findings provide important insights into the cellular alterations and molecular details in neuron development in E6AP-dependent ASD.

\section{Materials and Methods}

Antibodies, plasmids, and drugs. The following primary antibodies to the following proteins were used: mouse anti-E6AP [1:100 for immunocytochemistry (ICC) and immunohistochemistry (IHC), 1:1000 for Western blotting; Sigma-Aldrich, catalog \#8655], rabbit anti-cleaved caspase-3 (1:100 for ICC, 1:1000 for Western blotting; Cell Signaling Technology, catalog \#9661), rabbit anti-caspase-3 (1:1000 for Western blotting; Cell Signaling Technology, catalog \#9662), rabbit anti-XIAP (1:100 for ICC and IHC; Bioss Antibodies, catalog \#bs-1281r), rabbit anti-XIAP (1:1000 for Western blotting; Sigma-Aldrich, catalog \#PRS3331), mouse antiFLAG tag DYKDDDDK (1:1000 for Western blotting; Cell Signaling Technology, catalog \#8146), anti-GAPDH (1:5000 for Western blotting; EMD Millipore, catalog \#MAB374), mouse anti- $\alpha$ tubulin (1:2000 for Western blotting; Sigma-Aldrich, catalog \#T9026), rabbit anti-cleaved tubulin (Tub $\Delta$ Casp6; 1:500 for ICC, 1:5000 for Western blotting; provided kindly by Andrea LeBlanc at McGill University), mouse anti-NeuN (1:100 for ICC; EMD Millipore). The following secondary antibodies were used: IgG-HRP for Western blotting [1:5000; Bio-Rad, catalog \#170-6516 (mouse), catalog \#170-6515 (rabbit)], mouse Alexa Fluor 488 (1:500; Invitrogen, catalog \#A21121), rabbit Alexa Fluor 488 (1:500; Invitrogen, catalog \#A11094), mouse Alexa Fluor 555 (1:500; Invitrogen, catalog \#A21127), and rabbit Alexa Fluor 555 (1:500; Invitrogen, catalog \#A21428).

The following cDNA plasmids were obtained from Addgene: p4054E6AP (\#8658), E6AP C820A (\#37602), pEBB-XIAP (\#11558), and pCDNA3caspase-3 C163A (\#11814). mCherry-tubulin wild-type (WT) and mCherry-tubulin K40A were kind gifts from Dr. Saudou Frederic (Institut Curie).

pHR-pTRE-iCre-mCherry and pHR-rtTA (Tet-ON) were generously provided by Wilson Wong. To make pHR-pTRE-E6AP-mCherry, fulllength human E6AP was PCR amplified to include the restriction sites MluI and XmaI using the following oligonucleotides: 5' GCACGCGT GATGGAGAAGCTGCACCAG 3', 5' GTCCCGGGGCAGCATGCCAA ATCCTTT $3^{\prime}$. The same restriction sites were cut in pHR-pTRE-iCremCherry to remove iCre. E6AP PCR products were gel-purified (Qiagen, QIAquick Gel Extraction Kit) and subcloned into the vector. Similarly, pHR-pTRE-E6AP (without mCherry) was constructed using the restriction sites MluI and NotI and the following oligonucleotides: 5' GCACGCGTGATGGAGAAGCTGCACCAG 3' , 5' GTGCGGCCGCGT TACAGCATGCCAAATCCTTT 3'. To make AAV E6AP, E6AP was subcloned into AAV-ReaChR-citrine (Addgene, \#50954) using the BamHI and HindIII restriction sites.

Doxycycline (Sigma-Aldrich, catalog \#D9891) was used at $1 \mu \mathrm{g} / \mathrm{ml}$. MG132 was obtained from Sigma-Aldrich (catalog \#7449). The caspase-9 inhibitor III Ac-LEHD-CMK was obtained from EMD Millipore (catalog \#218728). Tubulin live-cell fluorescent labeling was done with the SiRTubulin Spirochrome probe (Cytoskeleton, catalog \#CY-SC002) according to the manufacturer's instructions.

Neuronal and human embryonic kidney cell culture and transfection. Primary cultured cortical and hippocampal neurons were prepared from embryonic day 18 rat embryos as previously described (Man et al., 2007; Hou et al., 2008). Briefly, dissociated neurons from rat hippocampus or cortex were seeded onto poly-L-lysine-coated (Sigma-Aldrich) coverslips in $60 \mathrm{~mm}$ dishes, each containing five coverslips, six-well plates, or glassbottom six-well plates (In Vitro Scientific). Neurons were maintained in Neurobasal medium (Thermo Fisher Scientific), supplemented with $2 \%$ Neurocult SM1 Neuronal Supplement (StemCell Technologies), 1\% horse serum (Atlanta Biologicals), 1\% penicillin/streptomycin (Corning), and L-glutamine (Corning). Seven days after plating, $5^{\prime}$-fluoro- $2^{\prime}$ deoxyuridine (10 $\mu \mathrm{M}$, Sigma-Aldrich) was added to the neuron media to suppress glial growth until experimental use. Human embryonic kidney (HEK) 293T cells were cultured in DMEM (Corning) supplemented with $10 \%$ heat-inactivated fetal bovine serum (Atlanta Biologicals), $1 \%$ penicillin/streptomycin, and L-glutamine. All cells were maintained in a humidified incubator at $37^{\circ} \mathrm{C}$ in an atmosphere containing $5 \% \mathrm{CO}_{2}$.

Transfection and viral infection. Neuron transfections were performed at days in vitro (DIV) 10-11 with Lipofectamine 2000 (Invitrogen), according to the manufacturer's instructions, with a DNA-to-Lipofectamine ratio 
of 2:1. Briefly, DNA and Lipofectamine were separately diluted in DMEM, then mixed together and incubated for $20 \mathrm{~min}$ at room temperature. The mixture was then added to neuron coverslips or glass-bottom plates and incubated for $4 \mathrm{~h}$ at $37^{\circ} \mathrm{C}$, after which the transfection medium was replaced with conditioned neuron medium. Neurons were fixed for ICC $24-36 \mathrm{~h}$ after transfection. HEK cell transfections were performed similarly at $60-70 \%$ cell confluency using the polyehtylenimine (PEI) transfection reagent (Polysciences) with a 3:1 PEI-to-DNA ratio. The cells were incubated with the transfection mixture for $8 \mathrm{~h}$, then rinsed twice with sterile PBS, and replaced with fresh HEK medium. HEK cells were lysed and collected $48 \mathrm{~h}$ after transfection.

Recombinant adeno-associated virus (AAV) was produced by transfecting HEK293T cells with the E6AP AAV or GFP AAV plasmid, along with viral packaging and envelope proteins XX6.80 and p50-Cap9 (AAV9) or XR2 (AAV2) using PEI. Three days following transfection, cells were lysed by freeze-thaw cycles and sonicated. The lysate was centrifuged at $3000 \times g$ for $30 \mathrm{~min}$ at $4^{\circ} \mathrm{C}$ and the supernatant was filtered through a $0.45 \mu \mathrm{m}$ filter and precipitated with PEG-it (Systems Biosciences). The mixture was centrifuged at $1500 \times g$ for $30 \mathrm{~min}$ and the resulting viral pellet was resuspended in $\mathrm{PBS}$ and aliquots were kept at $-80^{\circ} \mathrm{C}$. Primary neurons were infected with virus at DIV 2 and collected at DIV 12.

Animals. FVB/NJ-Tg(Ube3A)1Mpan/J mice (stock \#019730) and FVB/NJ WT mice (stock \#001800) were purchased from the Jackson Laboratory. All animals were maintained in accordance with guidelines of the Boston University Institutional Animal Care and Use Committee. To obtain Ube3A 2X Tg (2X Tg) animals, heterozygous males were mated with heterozygous females and homozygous animals were used for experiments. Both male and female pups were used for tissue collection.

ICC. Hippocampal neurons were washed twice in ice-cold ACSF and fixed for $10 \mathrm{~min}$ in a $4 \%$ paraformaldehyde/4\% sucrose solution at room temperature. Cell membranes were permeabilized for $10 \mathrm{~min}$ with $0.3 \%$ Triton X-100 (Sigma-Aldrich) in PBS, rinsed three times with PBS, then blocked with $5 \%$ goat serum in PBS for $1 \mathrm{~h}$. Following blocking, cells were incubated with primary antibodies made in $5 \%$ goat serum for $1 \mathrm{~h}$ at room temperature, washed with PBS, and incubated with Alexa Fluor-conjugated secondary antibodies for $1 \mathrm{~h}$. Cells were then mounted on microscopy glass slides with Prolong Gold anti-fade mounting reagent (Thermo Fisher Scientific, catalog \#P36930) for subsequent visualization.

$I H C$. For brain slices, animals were anesthetized in $\mathrm{CO}_{2}$ chamber and transcardially perfused with ice-cold PBS. The brains were removed and placed in $4 \%$ paraformaldehyde in PBS solution at $4^{\circ} \mathrm{C}$ for $4-6 \mathrm{~h}$, followed by incubation in a $30 \%$ sucrose PBS solution at $4^{\circ} \mathrm{C}$ for $24 \mathrm{~h}$. The brains were then placed in trays, submerged in optimal cutting temperature embedded medium (Tissue-Tek, catalog \#25608-930), and flash frozen by placing the trays in a dry-ice bath with methanol. Frozen brains were cut in $20 \mu \mathrm{m}$ sections on a Leica CM 1850 cryostat (Leica Biosystems) at $-20^{\circ} \mathrm{C}$. Slices were then rehydrated in PBS for $40 \mathrm{~min}$, followed by blocking and permeabilization in a $5 \%$ goat serum solution with $0.3 \%$ Triton X-100/PBS for $1.5 \mathrm{~h}$. Slices were then incubated with primary antibodies made in $5 \%$ goat serum overnight at $4^{\circ} \mathrm{C}$, washed three times with PBS, and incubated with secondary antibodies in $5 \%$ goat serum for $1 \mathrm{~h}$. Nuclei were stained with Hoechst, followed by three washes with PBS, and coverslipped with coverglass (Thermo Fisher Scientific, Fisherbrand \#12-544-D) with Prolong Gold. For GFP AAV2-infected brains, $100 \mu \mathrm{m}$ slices were made, rehydrated, and stained with Hoechst before coverslipping. These procedures were reviewed and approved by the Boston University Institutional Animal Care and Use Committee.

Golgi staining. Whole brains collected from transgenic animals at postnatal day $(\mathrm{P}) 15$ were subjected to Golgi neuron staining according to the manufacturer's instructions (FD Neurotechnologies, Rapid Golgistain Kit, catalog \#PK401). Brains were sliced in 200- $\mu$ m-thick slices using a cryostat. Stained slices were mounted on gelatin-coated microscopy slides (FD Neurotechnologies, catalog \#PO101) with Permount mounting medium (Thermo Fisher Scientific). Images obtained from Golgi-stained slices were traced using ImageJ for spines and NeuronJ for dendrites.

Microscopy. Hippocampal neurons mounted on glass slides were imaged with a Carl Zeiss inverted fluorescent microscope with a $40 \times$ or $63 \times$ oil-immersion objective and collected with AxioVision 4.5 software.
Golgi-stained brain slices were imaged with a $20 \times$ air objective. Images were quantified using National Institutes of Health ImageJ software.

Fixed brain sections from transgenic animals were imaged using a Zeiss LSM 700 laser scanning confocal microscope with a $25 \times$ oilimmersion objective. Images were collected as $4 \times 4$ tiles and stitched together using the Zen imaging software.

Live images of hippocampal neurons were obtained with a Zeiss LSM 700 Laser Scanning Confocal Microscope with a $63 \times$ oil-immersion objective in a temperature-controlled live-imaging chamber. Images of the same cells were obtained at several time points from induction of expression with the Zen imaging software.

Sholl analysis. Dendritic arborization was quantified using ImageJ. Original images of neurons were used to trace dendrites with the NeuronJ plugin. Using the Sholl analysis plugin, the center of the soma was used as a reference point and 10 concentric circles were made on the tracings: the starting radius was set to 35 pixels and the ending radius was set to 800 pixels (the outermost circle within the image). From these parameters, the number of intersections at each concentric circle was quantified and plotted.

Electrophysiology. Hippocampal neurons were transfected at DIV 12 with either surfGFP and pcDNA3.1 or surfGFP and E6AP. Two days following transfection, a coverslip of neurons was transferred to a recording chamber with the extracellular solution containing $140 \mathrm{~mm} \mathrm{NaCl}, 3$ mм KCl, $1.5 \mathrm{~mm} \mathrm{MgCl}_{2}, 2.5 \mathrm{~mm} \mathrm{CaCl}_{2}, 11$ glucose, and 10 HEPES, $\mathrm{pH}$ 7.4 , which was supplemented with tetrodotoxin $(1 \mu \mathrm{M})$ to block action potentials, 2-amino-5-phosphonopetanoate $(50 \mu \mathrm{M})$ to block NMDA receptors, and bicuculline $(20 \mu \mathrm{M})$ to block $\mathrm{GABA}_{\mathrm{A}}$ receptor-mediated inhibitory synaptic currents. Whole-cell voltage-clamp recordings were made with patch pipettes filled with an intracellular solution containing $100 \mathrm{mM}$ Csmethanesulfonate, $10 \mathrm{~mm} \mathrm{CsCl}, 10 \mathrm{~mm}$ HEPES, $0.2 \mathrm{~mm}$ EGTA, $4 \mathrm{~mm} \mathrm{Mg}$ ATP, $0.3 \mathrm{~mm}$ Na-GTP, $5 \mathrm{~mm}$ QX-314, and $10 \mathrm{~mm}$ Na-phosphocreatine, $\mathrm{pH}$ 7.4, with the membrane potential clamped at $-70 \mathrm{mV}$.

Immunoprecipitation. For ubiquitination immunoprecipitation assays, cells were rinsed with cold PBS and resuspended in $200 \mu \mathrm{l}$ of modified radioimmunoprecipitation assay (RIPA) lysis buffer [50 $\mathrm{mm}$ Tris-HCl, pH 7.4, $150 \mathrm{~mm} \mathrm{NaCl}, 1 \%$ NP-40 (Thermo Fisher Scientific), $1 \%$ sodium deoxycholate, and $1 \%$ SDS] with minicomplete protease inhibitor (Roche) and $20 \mu \mathrm{M} \mathrm{N}$-ethylmaleimide (Sigma-Aldrich). Lysates were further solubilized by sonication and volumes were adjusted to $500 \mu \mathrm{l}$ with more RIPA buffer. Protein A Sepharose beads (Santa Cruz Biotechnology) were added to the lysates along with antibodies against either FLAG or XIAP and samples were incubated overnight for $12-16 \mathrm{~h}$ on rotation at $4^{\circ} \mathrm{C}$. Immunocomplexes were washed three times with cold RIPA buffer, resuspended in $2 \times$ Laemmli buffer, and denatured at $95^{\circ} \mathrm{C}$ for $10 \mathrm{~min}$ before being subjected to Western blotting.

Sample collection. Brains were collected from transgenic animals at various developmental stages ( $\mathrm{P} 0-\mathrm{P} 40)$ and the hippocampus was dissected out. Brain tissues were either processed immediately or frozen at $-80^{\circ} \mathrm{C}$ for later processing. Tissues were lysed in RIPA buffer with $0.1 \%$ SDS by trituration, followed by brief sonication, and incubated for $1 \mathrm{~h}$ on a rotator at $4^{\circ} \mathrm{C}$. Samples were then centrifuged at $13,000 \mathrm{rpm}$ for $30 \mathrm{~min}$ at $4^{\circ} \mathrm{C}$ and the supernatant was collected. Protein levels were quantified by BCA assay (Pierce) and normalized to the same total protein concentration.

Western blotting. Cell lysates or immunoprecipitates were separated by SDS-PAGE, transferred to PVDF membranes, and probed with the appropriate antibodies. Immunointensity of Western blots was measured using ImageJ; values were normalized to corresponding tubulin or GAPDH inputs, and then normalized to controls where appropriate before statistical analysis.

Experimental design and statistical analyses. Both male and female mice were used for tissue collection. For developmental time points and brain lysate Western blots (see Figs. $6 A, B, 7 A-D$ ), three WT and three $2 \mathrm{X} \mathrm{Tg}$ mice were collected from 10 separate litters. For IHC (see Figs. 6, 6-1, available at https://doi.org/10.1523/JNEUROSCI.1511-17.2017.f6-1), two animals of each genotype were collected and 10 slices from each animal were used for each staining. For Golgi staining, two brains of each genotype were collected, five slices were made, and spines from 10 different neurons were analyzed. Dendritic morphology measurements were collected from 12 neurons from the same five slices. 
All values are reported as mean \pm SEM. A Student's $t$ test or two-way ANOVA with Tukey's post hoc test, Dunnett's multiple-comparison test, or Holm-Sidak test was used as appropriate. Statistical analysis was performed using Graphpad Prism.

\section{Results \\ E6AP overexpression leads to a reduction in dendritic arborization}

To investigate whether E6AP has any role in neuron morphogenesis, we overexpressed E6AP together with surface GFP in cultured hippocampal neurons at DIV 11 and observed their morphology at DIV 12. Twenty-four hours after transfection, increased levels of E6AP expression were detected in both the soma and the dendrites of neurons (Fig. 1-1, available at https:// doi.org/10.1523/JNEUROSCI.1511-17.2017.f1-1). At this stage, control neuron morphology shows multiple primary dendritic branches deriving from the soma, with elaboration of secondorder and third-order branches. Primary branches are distributed approximately evenly around the soma, often with one major, dominant dendrite (Fig. 1A). Surprisingly, compared with the GFP-only control, E6AP-transfected neurons revealed a marked reduction in dendritic morphology. Typically, a large portion of the dendrites disappeared, leaving only one or two major primary branches with multiple short minor neurites at the soma region (Fig. 1A). Sholl analysis showed that the dendritic branch numbers were significantly decreased along the distance from the soma $\left(F_{(1,198)}=55.44, p<0.0001\right.$, ANOVA; Fig. $1 B)$. The total number of dendrites and total dendritic length were also significantly reduced in E6AP-transfected neurons (branch number: control, $41.6 \pm 3.3$; E6AP, $17.5 \pm 1.41, p=$ $1.8 \times 10^{-5}$; total length: control, $2299.9 \pm 176.3 \mu \mathrm{m}$; E6AP, $877.4 \pm 78.1 \mu \mathrm{m}, p=1.4 \times 10^{-9} ; n=40$ cells per condition; Fig. $1 C)$. To further characterize the changes in dendritic morphology, we analyzed the branching pattern based on branching orders (Fig. $1 D)$. We found that although the length of primary branches was similar between both the control and E6AP conditions (control, $294.4 \pm 50.4 \mu \mathrm{m}, n=10$; E6AP, $231.1 \pm 37.7 \mu \mathrm{m}, n=10, p=$ 0.384 ), the secondary and tertiary branches were significantly shortened by E6AP overexpression (secondary branch: control, $378.6 \pm$ $37.8 \mu \mathrm{m}, n=10$; E6AP, $207.3 \pm 29.9 \mu \mathrm{m}, n=10, p=0.0086$; tertiary branch: control, $442.4 \pm 101.3 \mu \mathrm{m}, n=10$; E6AP, $60.58 \pm$ $12.5 \mu \mathrm{m}, n=10, p=0.0045$; Fig. $1 E$ ). These data showed that overexpressing E6AP leads to a reduction in dendritic arborization complexity in primary hippocampal neurons.

\section{Overexpression of E6AP in primary hippocampal neurons decreases the density of mature spines}

E6AP overexpression caused a drastic reduction in dendrite branching and length. We next wanted to know whether E6AP also plays a role in the regulation of dendritic spines. One possibility is that, as a result of reduced dendritic arborization, the remaining dendrites may have an increased density of spines to compensate for the loss of dendrites. To examine this, we transfected neurons at DIV 11 with E6AP and surface GFP (surfGFP), which contains a membrane attachment motif and is thus able to clearly delineate the minor membranous structures, such as the spines (Kameda et al., 2008; Fig. 1F). Twenty-four hours after transfection, neurons were fixed and spines were counted on 50 $\mu \mathrm{m}$ segments along primary dendrites. Mean spine density decreased in E6AP-overexpressing neurons (control, $0.84 \pm 0.09$ spines $/ \mu \mathrm{m}, n=10$ cells; E6AP, $0.27 \pm 0.02$ spines $/ \mu \mathrm{m}, n=10$ cells; $p=0.047$; Fig. $1 G$ ). Upon further characterization of the subtypes of spines, we found that E6AP neurons had fewer mushroom-type spines (control, $21.6 \pm 3.3 \%, n=10$; E6AP, $7.4 \pm 1.9 \%, n=10, p=0.007$; Fig. $1 H$ ). Conversely, the percentage of filopodia increased in E6AP neurons (control, $6.8 \pm 3 \%$, $n=10$; E6AP, $20.2 \pm 6.4 \%, n=10, p=0.047$; Fig. $1 H$ ). The percentages of stubby spines and thin spines were not significantly different between control and E6AP (Fig. $1 H$ ). Consistent with these findings, electrophysiological recordings revealed a decrease in the frequency, but not amplitude, of AMPA receptormediated miniature EPSCs (mEPSCs) in E6AP-transfected hippocampal neurons (control, $3.19 \pm 0.6 \mathrm{~Hz}, n=10$; E6AP, $1.16 \pm$ $0.23 \mathrm{~Hz}, n=10, p=0.0054$; Fig. 1-2, available at https://doi.org/ 10.1523/JNEUROSCI.1511-17.2017). The decrease in the number of mushroom spines and the increase in the number of filopodia in E6AP-transfected neurons, along with decreased frequency of mEPSCs, suggests a role for E6AP in spine maturation.

\section{E6AP overexpression causes active dendrite elimination}

We wanted to determine whether the change in dendritic morphology resulted from an inhibition in growth or from active removal of existing dendritic branches. To this end, hippocampal neurons were transfected at DIV 10 with surfGFP, and fixed after $24 \mathrm{~h}$ at DIV 11 for morphological analysis (Fig. 2A,B). At DIV 11, another set of neurons were transfected with either surfGFP only or together with E6AP, and fixed $24 \mathrm{~h}$ later at DIV 12. At DIV 11, the hippocampal cultured neurons already have elaborate dendritic arborization (Fig. 2A). If E6AP simply suppresses dendrite growth, arborization of E6AP-expressing neurons at DIV 12 is expected to be similar to that of the DIV 11 control neurons. However, compared with the surfGFP control neurons fixed at DIV 11, overexpressing E6AP from DIV 11 to 12 still led to a reduction in dendritic arborization compared with the DIV 11 neurons $\left(F_{(2,297)}=101.8, p<0.0001\right.$, ANOVA; significant difference in number of intersections at $40 \mu \mathrm{m}, p<0.0001 ; 55 \mu \mathrm{m}$, $p<0.0001 ; 86 \mu \mathrm{m}, p<0.0001 ; 101 \mu \mathrm{m}, p<0.0001 ; 116 \mu \mathrm{m}, p=$ $0.0129 ; 132 \mu \mathrm{m}, p=0.0023 ; 147 \mu \mathrm{m}, p=0.0008$; $n=10$, Dunnett's multiple-comparison test; Fig. $2 C$ ). This suggests that the E6AP-induced downregulation in dendrite branching was not likely due to an inhibition of dendrite growth, but rather stemmed from an active removal of existing dendritic branches.

To further examine the cellular process leading to reduced dendritic complexity, we performed live imaging with inducible E6AP expression. We first tested our tetracycline-inducible E6AP for its ability to change dendrite morphology. Indeed, expression of pTRE-E6AP-mCherry (pTRE-E6AP-mCh) after doxycycline $(1 \mu \mathrm{g} / \mathrm{ml})$ treatment caused significant dendrite reduction within $24 \mathrm{~h}$. By comparison, no dendrite reduction was seen among untreated pTRE-E6AP-mCh cells $\left(F_{(1,528)}=25.08, p<0.0001\right.$, ANOVA; Fig. 2-1A,C, available at https://doi.org/10.1523/ JNEUROSCI.1511-17.2017.f2-1). Additionally, expression of the control pTRE-mCh did not lead to changes in morphology with or without doxycycline treatment (Fig. 2-1 $A, B$, available at https://doi.org/10.1523/JNEUROSCI.1511-17.2017.f2-1). To study the molecular process of E6AP-dependent dendritic remodeling, hippocampal cultures on a glass-bottom plate were transfected with surfGFP together with either pTRE-mCh (control) or pTRE-E6AP-mCh. One day after transfection, when neuron structures became clearly visible with surfGFP, doxycycline was added to the medium to induce E6AP expression. Neurons were then imaged every $6 \mathrm{~h}$ for the next $24 \mathrm{~h}$. In the control neurons, we found some minor dendrite growth and retraction, but the overall structure remained stable (Fig. $2 D$, top row). In contrast, the dendritic arbors of the E6AP-expressing neurons changed drastically over the same period. Many pre-existing den- 
A

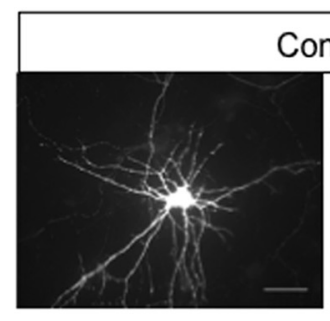

Control

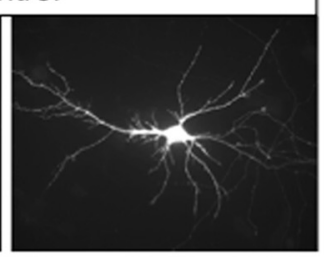

B

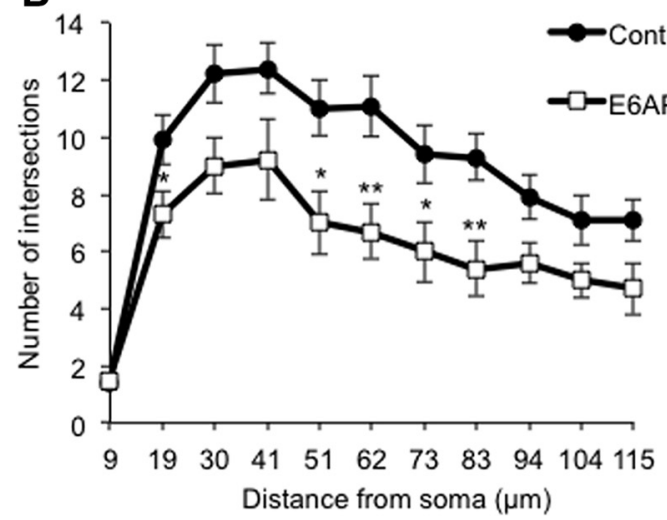

D

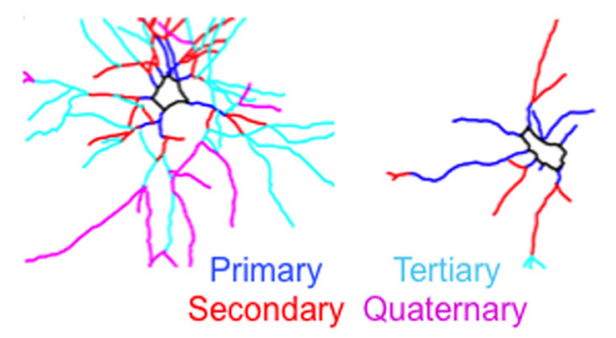

F

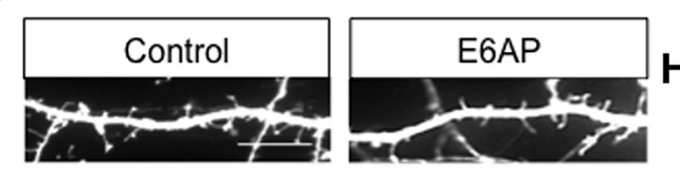

G

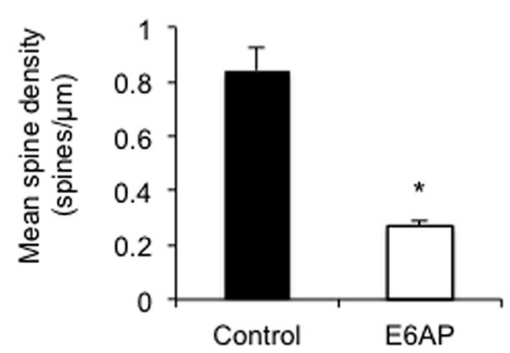

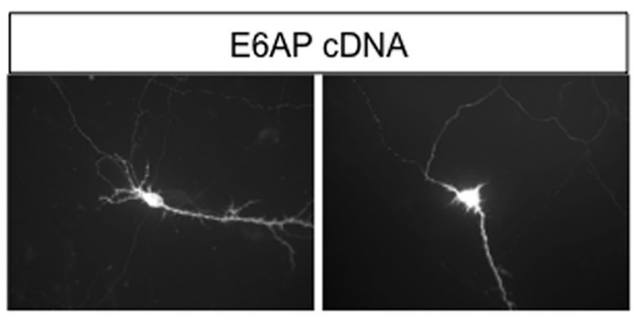

C

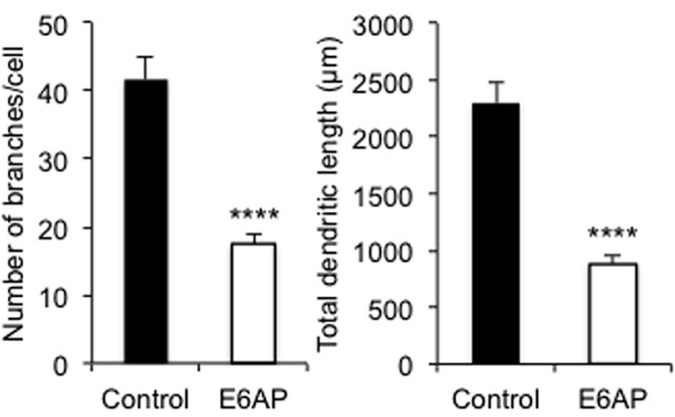

E

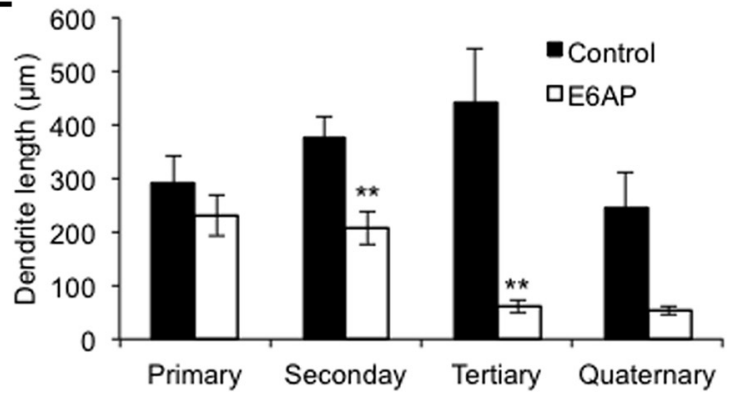

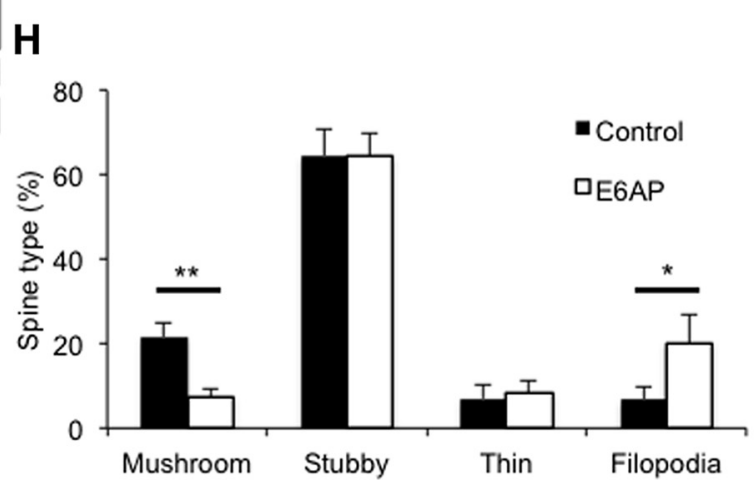

Figure 1. E6AP overexpression reduces the complexity of dendritic arborization. $A$, Hippocampal neurons were transfected with surfGFP together with vector CDNA (Control) or E6AP CDNA at DIV 11 and imaged for morphology $24 \mathrm{~h}$ after transfection. Scale bar, $50 \mu \mathrm{m}$. $\boldsymbol{B}$, Sholl analysis of dendritic branch numbers. Overexpression of E6AP resulted in a decrease in dendritic complexity; $n=$ 40 neurons for each condition. C, Total dendritic branch number and total dendritic length were reduced in E6AP-transfected neurons; $n=40$. D, E, Dendrites were characterized as either primary, secondary, tertiary, or quaternary based on their arborization pattern. Representative images of neurons were traced with primary dendrites in blue, secondary in red, tertiary in cyan, and quaternary in magenta. E6AP overexpression led to a decrease in secondary and tertiary dendritic branch length; $n=10 . F$, Images of dendritic spines from neurons transfected at DIV 11 with surfGFP or together with E6AP for $24 \mathrm{~h}$. Scale bar, $10 \mu \mathrm{m}$. G, Mean spine density decreased in E6AP neurons; $n=10$ cells. $\boldsymbol{H}$, Spines were categorized as either mushroom, stubby, thin, or filopodia. Increased E6AP expression led to a decrease in mushroom-type spines and an increase in filopodia; $n=10$ cells. Error bars represent SEM, ${ }^{*} p<0.05,{ }^{* *} p<0.01,{ }^{* * * *} p<0.0001$ (Fig. 1-1, available at https://doi.org/10.1523/JNEUROSCI.1511-17.2017.f1-1; cellular distribution of E6AP in hippocampal neurons; Fig. 1-2, available at https://doi.org/10.1523/JNEUROSCI.1511-17.2017.f1-2; overexpression of E6AP causes reduction in $\mathrm{mEPS}(\mathrm{s})$.

drites were removed, while a smaller number of neurites either grew or remained stable (Fig. $2 D$, middle row). Compared with control, E6AP neurons had more retraction events (control, $35.5 \pm 7.8 \%, n=9$; E6AP, $49.09 \pm 5.2 \%, n=7, p<0.05$; Fig. $2 E)$ and a greater percentage of overall dendrite reduction (control, $17.57 \pm 4.6 \%, n=9$; E6AP, $39.4 \pm 6.8, n=7, p=0.022$; Fig. $2 F$ ), indicating elevated activity in dendritic remodeling. The total length of growth was not significantly different in E6AP neurons, 
A
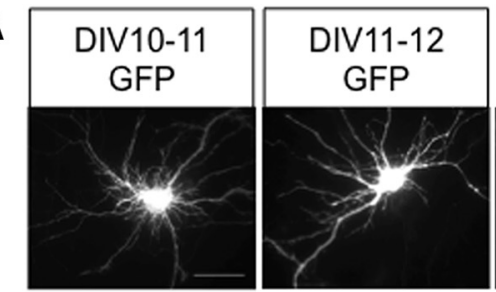

B
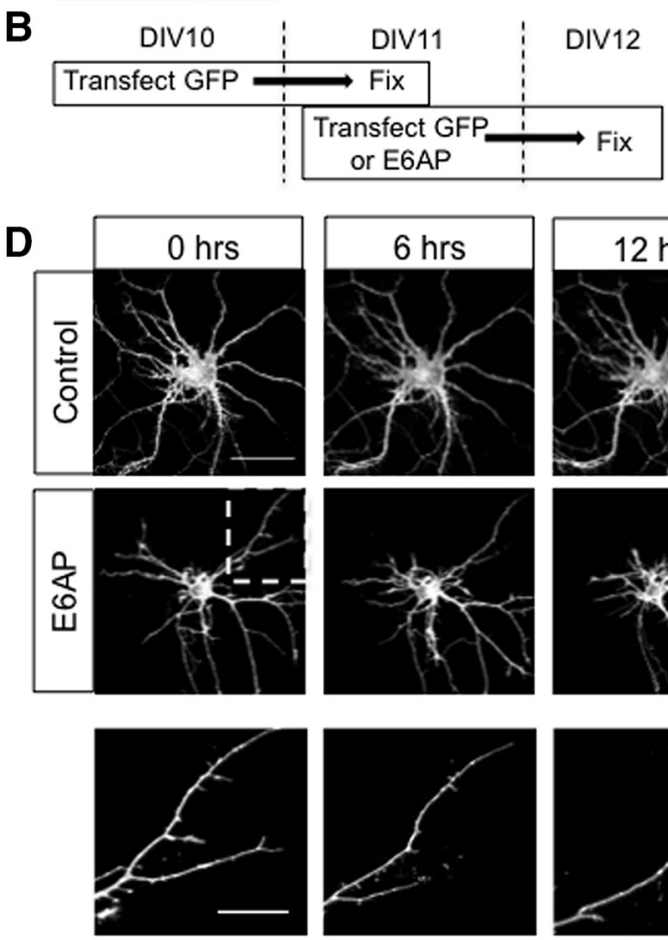

E

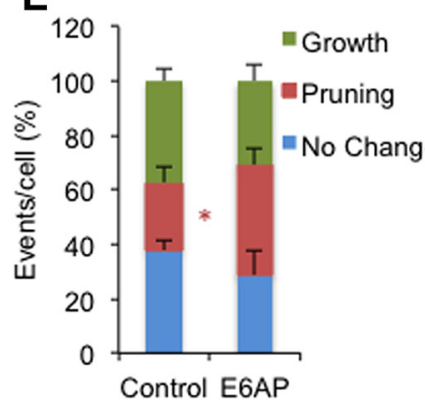

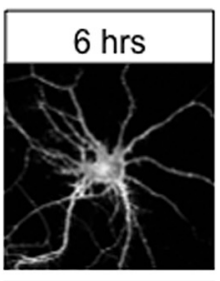
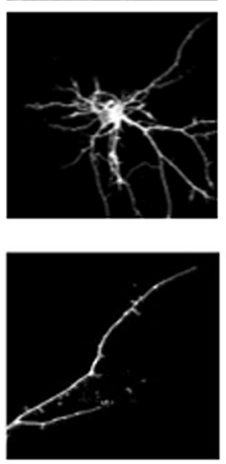

$\mathbf{F}$

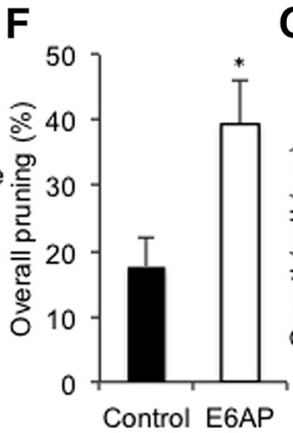

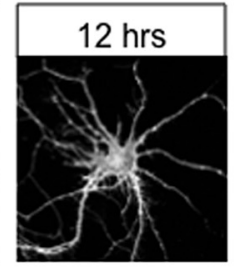
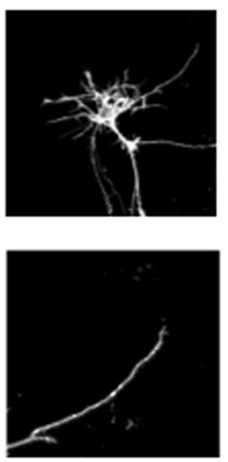

G
C
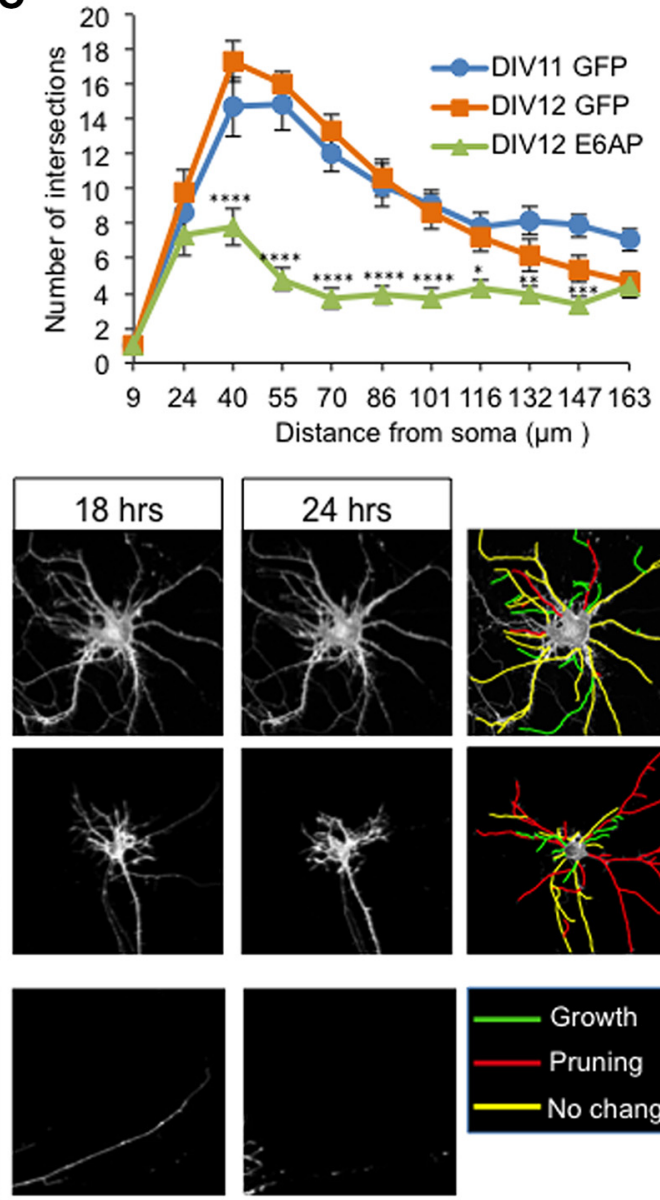

H
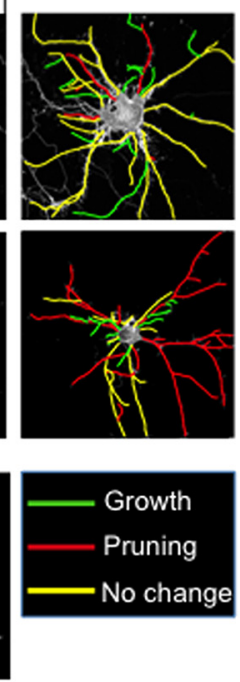
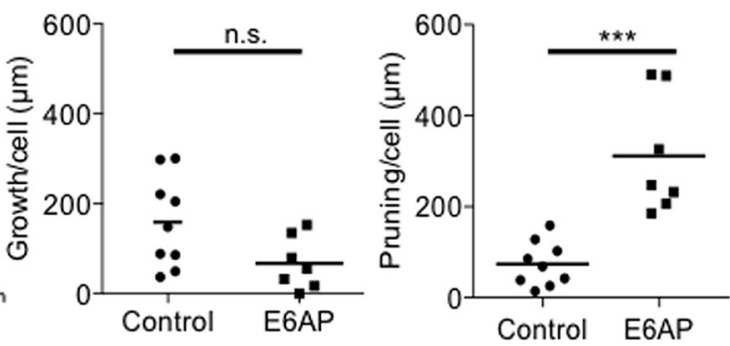

Figure 2. E6AP overexpression triggers active dendrite retraction and elimination. $\boldsymbol{A}, \mathrm{GFP}$ images of neurons transfected from DIV 10 to 11 or from DIV 11 to 12 . Scale bar, $50 \mu \mathrm{m}$. $\boldsymbol{B}$, Diagram of the experimental design. C, Sholl analysis showed reduced dendritic arborization in E6AP neurons at DIV 12 compared with either DIV 11 or 12 control cells; $n=10$ cells per condition. D, DIV 11 hippocampal neurons were transfected with pTRE-mCherry (Control) or pTRE-E6AP-mCherry (E6AP) and their expression was induced by the addition of doxycycline (Dox, $1 \mu \mathrm{g} / \mathrm{ml}$ ) $24 \mathrm{~h}$ after transfection (Fig. 2-1, available at https://doi.org/10.1523/JNEUROSCI.1511-17.2017.f2-1). Live imaging was performed immediately after Dox treatment (time 0) and every $6 \mathrm{~h}$ for 24 h. Colored tracings represent dendrites that increased in length (green), decreased in length (red), or remained the same (yellow). A portion of dendrites was enlarged (bottom row) to show dendrite retraction and fragmentation in E6AP neurons. Scale bar, $50 \mu \mathrm{m}$. Scale bar for bottom inset, $10 \mu \mathrm{m}$. $\boldsymbol{E}$, Analysis of live-imaging dendritic events. A significant increase in retraction events was detected in E6AP-expressing neurons compared with the control neurons. $\boldsymbol{F}$, Percentage of total length of retracted dendrites at $24 \mathrm{~h}$. $\boldsymbol{G}$, Total length of dendritic growth after $24 \mathrm{~h}$. $\boldsymbol{H}$, Total length of dendritic retraction after $24 \mathrm{~h} ; n=9$ cells for control and $n=7$ cells for E6AP for live-imaging experiments. Error bars represent SEM, ${ }^{*} p<0.05,{ }^{* *} p<0.01,{ }^{* * *} p<0.001,{ }^{* * * *} p<0.0001$.

supporting the notion that the reduction in dendritic arbor complexity is not a result of inhibition of growth (Fig. 2G). The total length of retracted branches significantly increased in E6AP cells (control, $73.8 \pm 25.4 \mu \mathrm{m}, n=9$; E6AP, $310.9 \pm 62.8 \mu \mathrm{m}, n=7$, $p=0.0002$; Fig. $2 H$ ), suggesting that the dendritic tree of E6AP neurons is reduced by active retraction. Interestingly, we observed that the dendrites in the process of remodeling showed two typical structural changes: distal thinning and fragmentation (Fig. 2D, bottom row). Some dendrites were found to be thinning at the distal section close to the tip and shrinking before complete elimination, whereas others were found to disintegrate into fragments at the tip of the neurite and gradually break down before disappearing.

Caspase-3 activity is required for E6AP-induced dendritic remodeling

We next wanted to know the underlying molecular cascade leading to E6AP-induced dendritic withdrawal. Caspases are a family of cysteine proteases that play a key role in the signaling cascade 
A
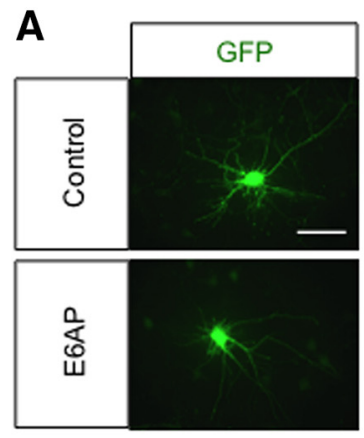

B

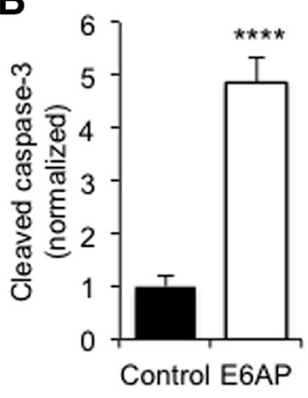

F

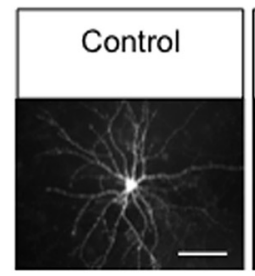

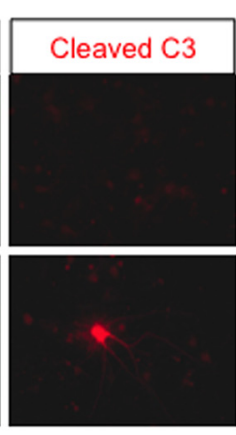

C $\quad$ AAV9 AAV9 GFP EGAP
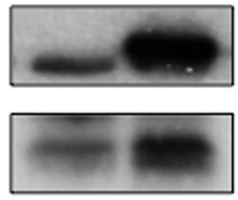

E6AP

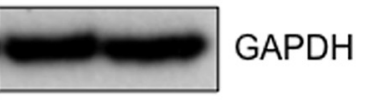

D

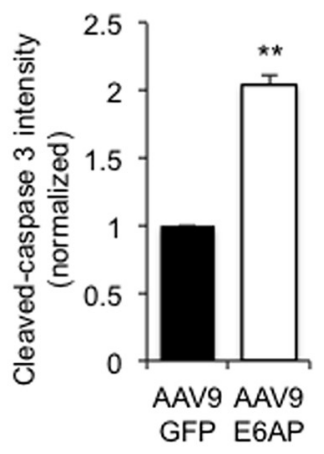

E

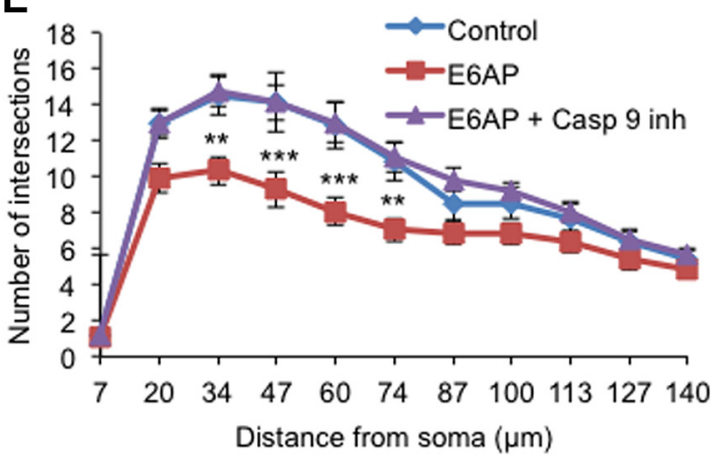

G
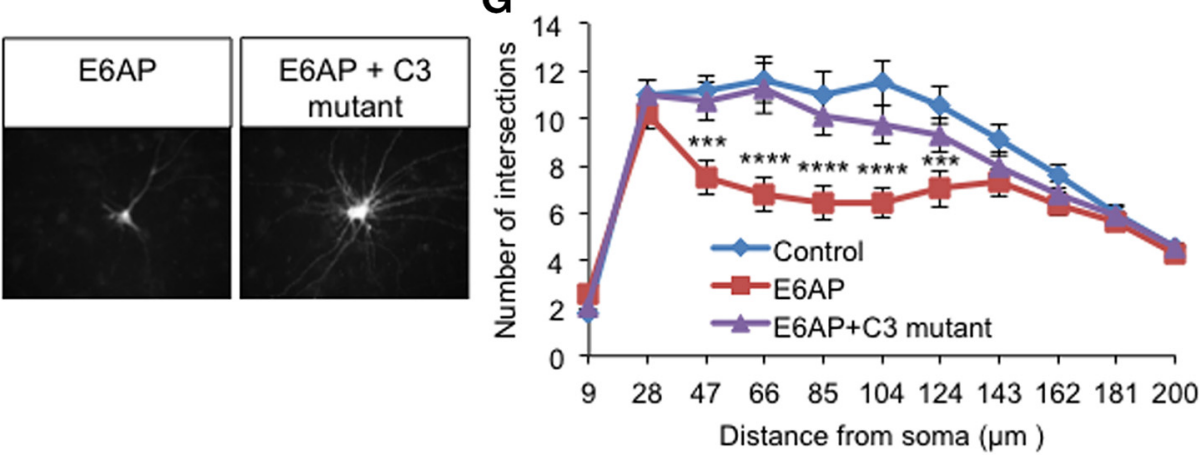

Figure 3. Activation of caspase-3 is required for E6AP-dependent dendritic remodeling. $\boldsymbol{A}$, Neurons were transfected with surfGFP (green; Control) or together with E6AP, and the cleaved (activated) caspase-3 (red) was immunostained $24 \mathrm{~h}$ later. Scale bar, $50 \mu \mathrm{m}$. B, Quantification of the cleaved caspase-3 immunofluorescence signals; $n=10$. E6AP expression resulted in higher levels of cleaved caspase-3. C, D, DIV 2 hippocampal neurons were infected with AAV 9 GFP virus or AAV9 E6AP virus for 10 d and cleaved caspase-3 levels were measured by Western blot. Neurons infected with E6AP virus showed higher levels of cleaved caspase-3; $n=3$ independent experiments. $\boldsymbol{E}$, Dendritic arborization reduction in E6AP neurons was blocked by inhibiting caspase-3 cleavage with the caspase-9 inhibitor Ac-LEHD-CMK (150 nM) at the time of transfection, as shown by Sholl analysis; $n=10 . \boldsymbol{F}, \mathbf{G}$, Neurons were transfected with surfGFP (control) or together with E6AP or E6AP + Casp3 C163A (E6AP + C3 mutant), a catalytic caspase-3 mutant. Scale bar, $50 \mu \mathrm{m}$. Sholl analysis revealed a rescue of the E6AP-induced dendritic remodeling by Casp3 C163A; $n=$ 10. Error bars represent SEM, ${ }^{* *} p<0.01,{ }^{* * *} p<0.001,{ }^{* * *} p<0.0001$.

involved in apoptosis, differentiation, and neuromorphogenesis (Unsain and Barker, 2015). An activated caspase cleaves and activates downstream caspases, leading to digestion of target functional proteins and resulting in wide-ranging cellular destruction as well as functional modification. In addition to global activation leading to cell death, activation of the caspase cascade has been found to occur locally at restricted regions in neurons ( $\mathrm{Li}$ et al., 2010). Local caspase activation is required for regulating axonal branching in retinal ganglion cells (Campbell and Okamoto, 2013), and developmental degeneration of dorsal root ganglion neurons necessitates caspase activity (Unsain et al., 2013). In addition, efficient axon regeneration and growth following an injury also involves the activation of the caspase cascade (Verma et al., 2005). In cultured hippocampal neurons, caspase activity has been shown to be required for spine dynamics (Jiao and Li, 2011; Ertürk et al., 2014). Consistently, caspases also play an important role in synaptic plasticity (Li et al., 2010). As these studies highlight the important role of caspases in morphological changes relevant to neuronal development, we wondered whether this pathway is also involved in E6AP-dependent dendritic reorganization. To this end, we first measured caspase- 3 activity in neurons overexpressing E6AP, as caspase- 3 is a crucial downstream executioner caspase in the cascade. Hippocampal neurons were transfected with surfGFP alone or together with E6AP at DIV 11 and fixed $24 \mathrm{~h}$ later. When neurons were immunostained with an antibody specifically against cleaved caspase-3 (activated form), we found that the E6AP-transfected neurons had a 4.8-fold increase in cleaved caspase- 3 levels compared the control $(n=10$, $p=8.1 \times 10^{-6}$; Fig. $3 A, B$ ). To further confirm the effect of E6AP on caspase- 3 activation, we infected neurons with AAV9 GFP or AAV9 E6AP virus for $10 \mathrm{~d}$. Consistent with the immunostaining results, Western blotting showed that cleaved caspase- 3 lev- 
els were significantly increased in E6AP-infected neurons (2.04 \pm 0.07-fold normalized to control, $n=3, p=0.005$; Fig. $3 C, D)$.

We then wanted to investigate whether the caspase pathway is involved in E6AP-dependent dendritic remodeling. In hippocampal neurons transfected with E6AP, we suppressed caspase-3 activation by the application of Ac-LEHD-CMK (150 nM), an irreversible inhibitor of caspase-9, which is an upstream activator of caspase-3 (Mocanu et al., 2000). Indeed, treatment with the caspase-9 inhibitor blocked the E6AP-dependent dendritic shrinking (Fig. $3 E$ ), indicating a requirement of caspase- 3 activity in E6AP-induced dendritic remodeling. To further confirm the role of caspase-3, we performed dominant-negative experiments. DIV 11 neurons were transfected with E6AP along with a caspase-3 catalytic mutant plasmid, Casp3 C163A. Consistent with the pharmacological treatment, overexpression of this mutant caspase- 3 abolished the E6AP-induced morphological changes (Fig. $3 F, G$ ), demonstrating a clear rescue of the remodeling phenotype by inhibition of caspase- 3 cleavage and activity. These data strongly suggest that the caspase cascade, primarily caspase-3, plays a crucial role in E6AP-dependent dendritic remodeling in hippocampal neurons.

\section{E6AP as an E3 ligase targets XIAP for ubiquitination}

As a HECT E3 ligase, E6AP is expected to execute its effect by ubiquitination and subsequent degradation of target protein(s). Consistently, when we expressed the E6AP E3 ligase mutant E6AP C820A, the dendritic retraction effect was markedly suppressed (Fig. 4-1A,B, available at https://doi.org/10.1523/ JNEUROSCI.1511-17.2017.f4-1), confirming the role of E6AP E3 ligase activity in remodeling. Because E6AP expression led to an increase in caspase- 3 activity, we hypothesized that it may target an intermediate molecule that inhibits caspases, so that ubiquitination and degradation of such an inhibitory molecule would lead to caspase- 3 activation. In line with this thought, we found that the most likely candidate protein is the family of IAPs, the inhibitors of apoptosis. IAPs are the first identified family of endogenous cellular inhibitors of caspases in mammals; and members of that family, namely XIAP, c-IAP1, and c-IAP2, have been shown to potently bind to and inhibit caspase-3, caspase-7, and caspase-9 (Deveraux et al., 1997; Roy et al., 1997). Of these IAPs, XIAP is ubiquitously expressed in all adult and fetal tissues, including the brain (Rajcan-Separovic et al., 1996), whereas c-IAP1 and c-IAP2 are mainly expressed in the kidney, small intestine, liver, and lung, with only minimal expression in the CNS (Young et al., 1999). XIAP, therefore, was considered the top candidate as an E6AP target. To directly investigate whether XIAP is subject to ubiquitination by E6AP, we performed ubiquitination assays as reported in our earlier studies (Lin et al., 2011; Huo et al., 2015). HEK293T cells were transfected with HA-ubiquitin and FLAG-XIAP, together with E6AP WT or E6AP C820A. Two days after transfection, FLAG-XIAP was isolated by immunoprecipitation and probed for HA (ubiquitin). We found that compared with control, E6AP overexpression resulted in strong ubiquitination of XIAP ( $7 \pm 0.7$-fold increase, $n=3, p=$ 0.001 ; Fig. $4 A, B)$. In contrast, much reduced ubiquitination was detected in cells expressing the ligase dead E6AP C820A, indicating E6AP as an E3 ligase for XIAP ubiquitination (2.5 \pm 0.3 -fold increase, $n=3, p>0.05$; Fig. $4 A, B$ ). To further confirm the role of E6AP on XIAP ubiquitination, we performed a ubiquitination assay in neurons infected with either GFP or E6AP AAV9 virus for $10 \mathrm{~d}$. Indeed, compared with GFP, E6AP virus caused a significant increase in XIAP ubiquitination (2.85 \pm 0.12 -fold in- crease, $n=3, p=0.0001$; Fig. $4 C, D)$, further supporting XIAP as a ubiquitination target for E6AP.

\section{E6AP downregulates XIAP levels by ubiquitination-dependent degradation}

Following ubiquitination, the modified protein is usually sorted to the proteasome for degradation. To examine whether E6APdependent XIAP ubiquitination leads to its degradation, we immunostained XIAP in neurons transfected with either a control vector or E6AP. As expected, the endogenous XIAP intensity was markedly reduced in neurons overexpressing E6AP (40 \pm 9\%, $n=10, p=0.042$; Fig. $4 E, F)$. Whole-cell lysates of E6APoverexpressing HEK cells also had lower levels of XIAP, whereas XIAP levels in E6AP C820A cells were comparable to those of control cells (E6AP, $0.55 \pm 0.06, n=3, p=0.0017$; E6AP C820A, $1.11 \pm 0.1, n=3, p>0.05$; Fig. $4 A, B)$. To examine XIAP stability in neurons, we applied E6AP virus to hippocampal neurons for $10 \mathrm{~d}$ and probed XIAP by Western blotting. Indeed, the total protein level of XIAP was markedly reduced in E6AP virusinfected neurons ( $0.66 \pm 0.07, n=3, p=0.0037$; Fig. 4G,H), confirming that E6AP indeed causes a reduction in XIAP protein levels.

The E6AP-induced reduction in XIAP could result from facilitated degradation or inhibited protein synthesis. To clarify this, we performed degradation assays in HEK293 cells. Two days after transfection with XIAP alone or together with E6AP, HEK cells were incubated with the protein translation inhibitor cycloheximide for varied periods of time. Western blots showed that XIAP had an increased rate of degradation in cells with E6AP overexpression compared with the control $\left(F_{(1,40)}=\right.$ $16.68, p=0.0002$, ANOVA; at $4 \mathrm{~h}$ : control, $0.82 \pm 0.09$; E6AP, $0.41 \pm 0.13, n=4, p=0.0195$; at $6 \mathrm{~h}$ : control, $0.49 \pm 0.07$; E6AP, $0.16 \pm 0.06, n=4, p=0.025$; Fig. $4 I, J)$. This result confirmed that E6AP overexpression led to an elevated turnover rate for XIAP.

We predicted that if downregulation of XIAP and thus activation of caspase-3 mediates E6AP-dependent structural remodeling, then dendritic changes might be prevented by overexpression of XIAP. To this end, we transfected neurons with E6AP alone or together with XIAP, and Sholl analysis was performed $24 \mathrm{~h}$ later. We found that while E6AP-transfected neurons had marked dendritic remodeling $\left(F_{(2,297)}=16.45, p<0.0001\right.$, ANOVA $)$, no significant changes in dendritic arborization were detected in cells cotransfected with E6AP and XIAP (Fig. $4 K, L$ ). Thus, consistent with the role of E6AP-mediated ubiquitination and degradation of XIAP, these results strongly indicate a reduction in XIAP as a key step in E6AP-dependent dendritic remodeling.

\section{The cytoskeletal component tubulin is targeted by caspases in E6AP-dependent dendritic remodeling}

Having implicated the involvement of caspases in E6APdependent dendritic remodeling, we wanted to examine the subcellular events that lead to the dendritic remodeling under E6AP overexpression. As microtubules are essential for structural integrity of dendrites in neurons, we wondered whether microtubule integrity was compromised during E6AP-dependent dendritic retraction. In our examination of dendritic branches under active remodeling, live imaging revealed a destabilization, disintegration, and retraction from the distal tip of a branch (Fig. 2D). To determine whether changes in microtubules occurred during E6AP-induced dendrite retraction, we performed live-imaging experiments by live-labeling microtubules. We transfected neurons on glass-bottom dishes with surfGFP and tet-inducible 


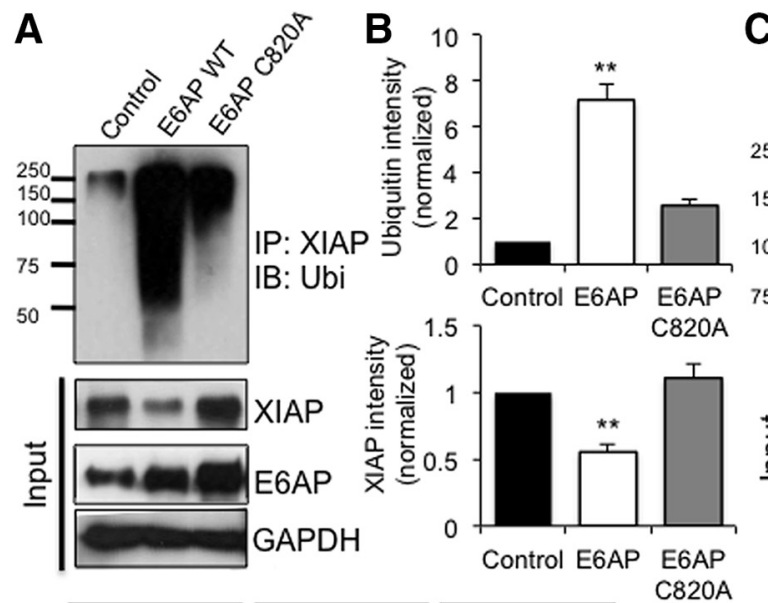

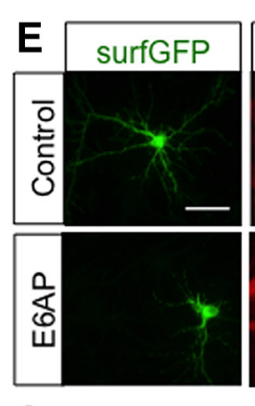

I

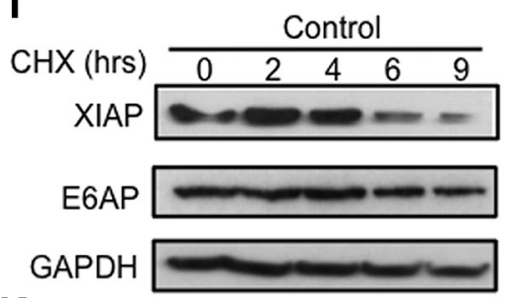

K
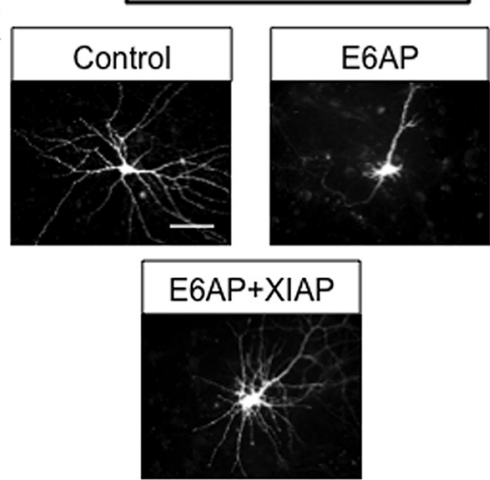

C

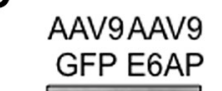

D
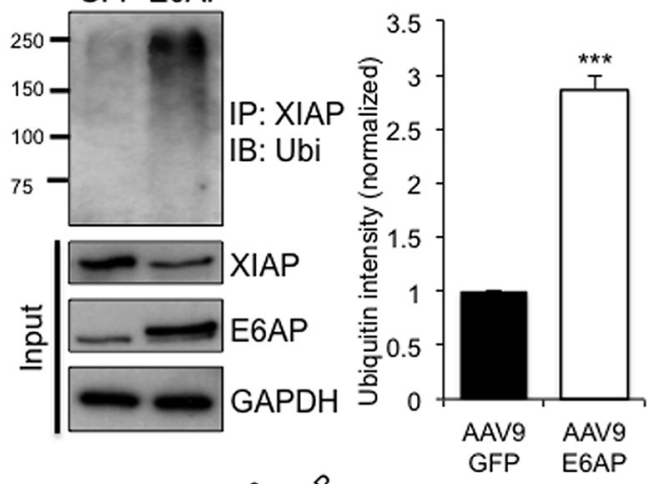

\section{H}

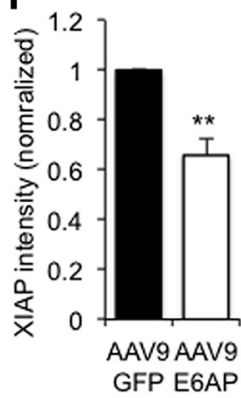

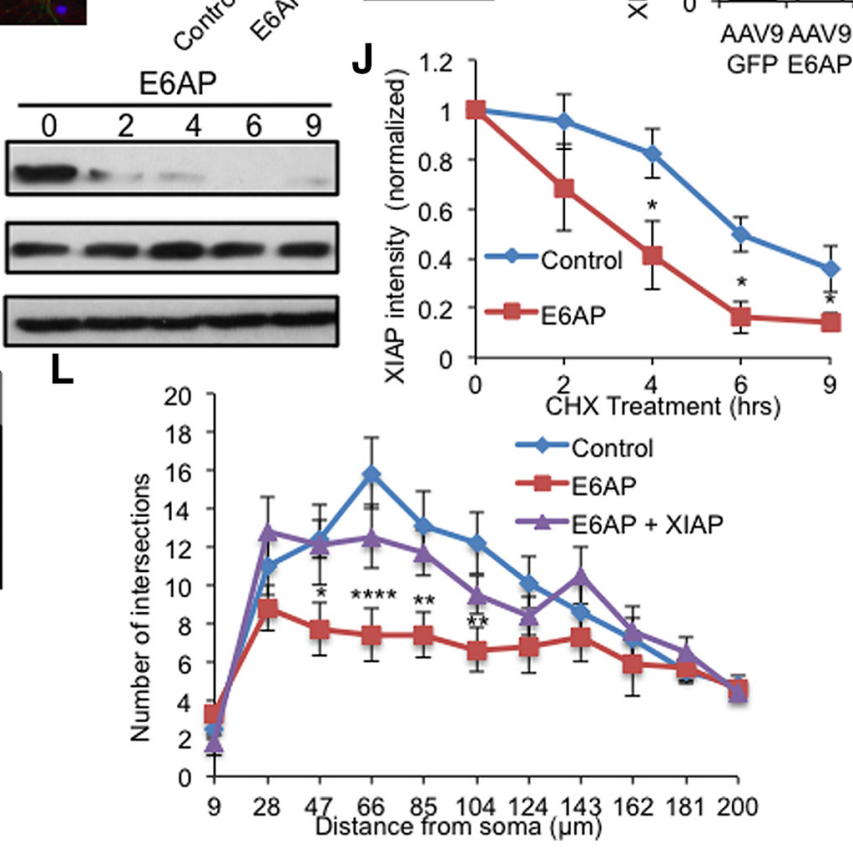

Figure 4. E6AP targets XIAP for ubiquitination and degradation. A, XIAP ubiquitination assay. HEK293 cells were transfected with FLAG-XIAP, HA-ubiquitin, and either a vector control, E6AP, or the E3 ligase dead mutant E6AP (820A for $2 \mathrm{~d}$. XIAP was immunoprecipitated and probed for ubiquitin (ubi). Cell lysates (input) were also probed to detect total protein levels. $B$, Quantification of Western blot intensities. E6AP, but not E6AP (820A, caused an increase in XIAP ubiquitination and a decrease in XIAP protein levels; $n=3$ independent experiments. $\boldsymbol{C}, \boldsymbol{D}$, XIAP ubiquitination assays using lysates of neurons infected with AAV9 GFP or AAV9 E6AP virus for $10 \mathrm{~d}$. Increased intensity of ubiquitination signals on XIAP was detected; $n=3$ independent experiments. $E$, Immunostaining of endogenous XIAP (red) in neurons transfected with surfGFP ( $g r e e n$ ) or together with E6AP. Nuclei were indicated by DAPI staining (blue). Scale bar, $50 \mu \mathrm{m}$. $\boldsymbol{F}$, Quantification of the XIAP signal intensity relative to the control; $n=10$ cells per condition. $\mathbf{G}, \boldsymbol{H}$, Neurons were infected with AAV9 GFP virus or AAV9 E6AP virus for $10 \mathrm{~d}$, and XIAP levels were measured by Western blot. Quantification showed a reduced level of XIAP in E6AP-infected neurons; $n=3$ independent experiments. I, Degradation assay of XIAP with or without E6AP. Transfected HEK cells were treated without cycloheximide (CHX) for various time points and cell lysates were collected to examine XIAP levels by Western blot. J, Quantification of the degradation rate of XIAP over time; $n=4$ independent experiments. $\boldsymbol{K}$, Morphology imaging of primary neurons transfected with surfGFP alone or together with E6AP or E6AP + XIAP. The effect on dendritic arborization markedly decreased in neurons expressing E6AP C820A (Fig. 4-1, available at https://doi.org/10.1523/JNEUROSCI.1511-17.2017.f4-1). Scale bar, $50 \mu \mathrm{m}$. L, Sholl analysis showing a blockade of the E6AP effect in dendritic remodeling by XIAP overexpression; $n=10$ cells per condition. Error bars represent SEM, ${ }^{*} p<0.05,{ }^{* *} p<0.01,{ }^{* * *} p<0.001,{ }^{* * * *} p<0.0001$.

pTRE-E6AP for $24 \mathrm{~h}$, and loaded neurons with the microtubule dye SiR-Tubulin before inducing E6AP expression with doxycycline. Images of the tubulin signal and the overall neuronal structure by surfGFP were captured every $20 \mathrm{~min}$ for $12 \mathrm{~h}$ (rep- resentative images taken at $7 \mathrm{~h}$ after doxycycline treatment). We found that during dendrite remodeling, microtubules showed thinning and shrinking at times before withdrawal of physical structure of a branch, as indicated by the retraction of the labeled 
A

0
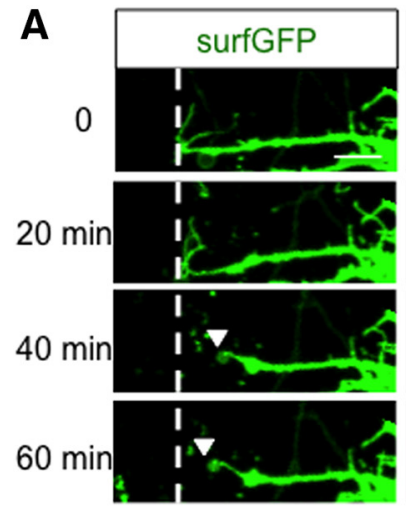

D
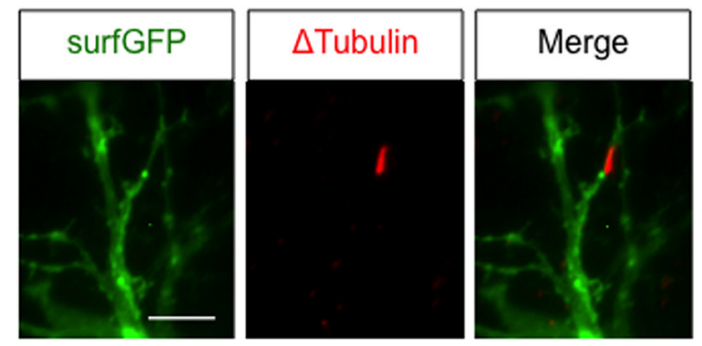

F
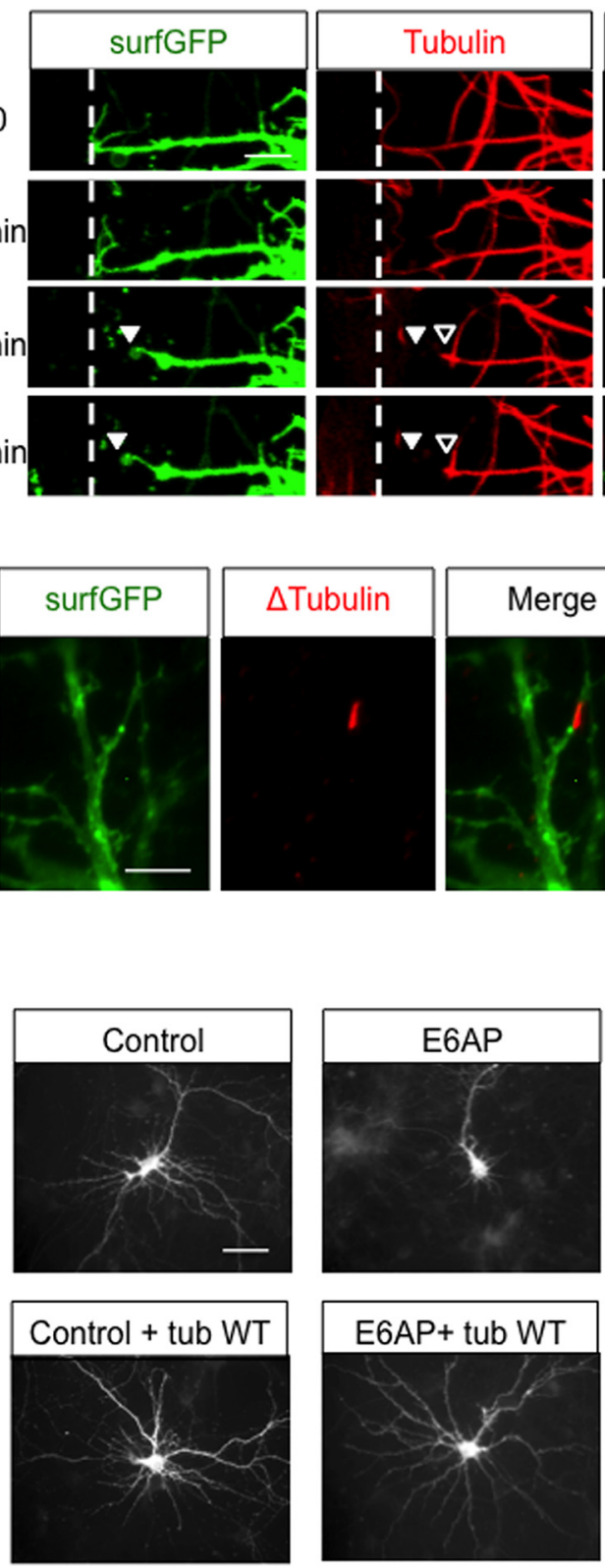

H

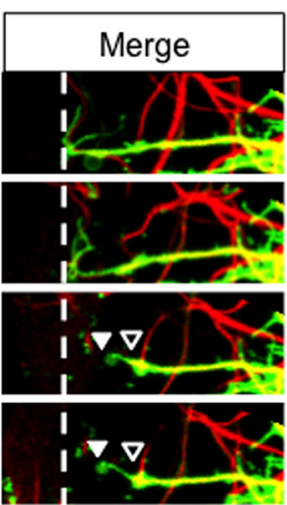

B
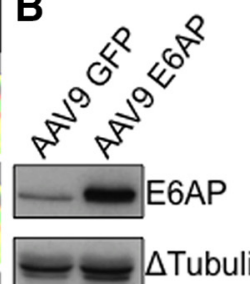

$\triangle$ Tubulin
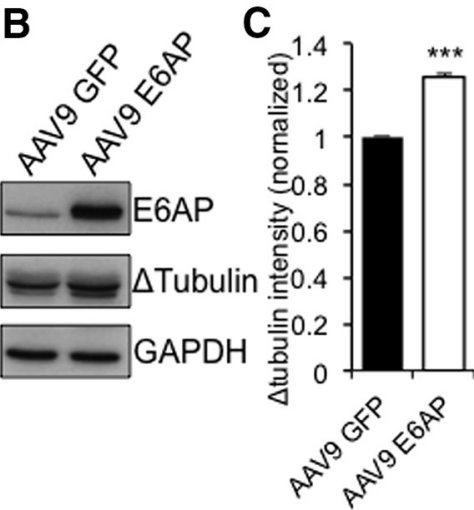

E

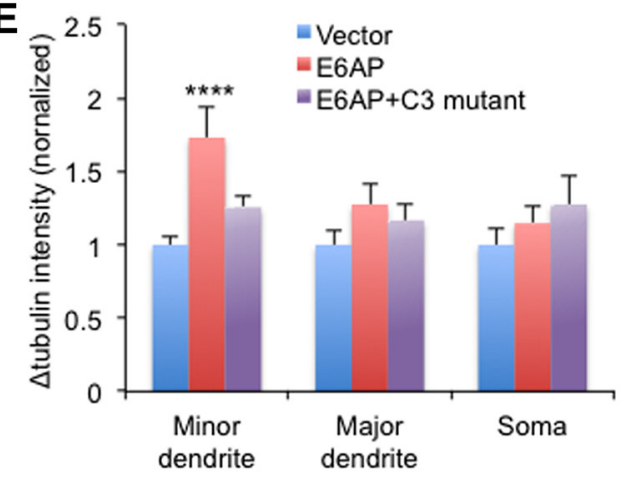

G

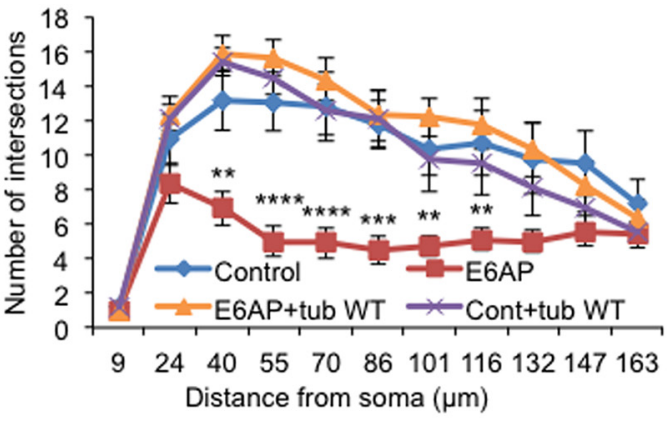
Distance from soma $(\mu \mathrm{m})$

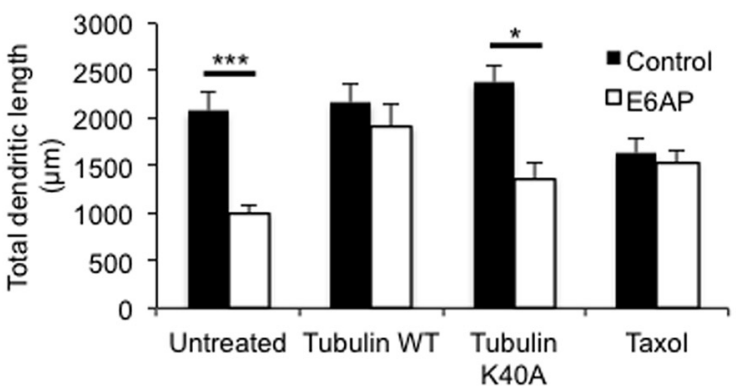

Figure 5. Microtubule cleavage and retraction in E6AP-induced dendritic remodeling. $\boldsymbol{A}$, Neurons were transfected with surfGFP and pTRE-E6AP for $24 \mathrm{~h}$, and loaded with SiR-Tubulin, a fluorogenic and cell-permeable dye for tubulin labeling, before being treated with doxycycline (Dox) to induce E6AP expression. Tubulin and surfGFP images were obtained every 20 min for $12 \mathrm{~h}$ following Dox application. Representative images show that retraction of microtubule (red; hollow arrowhead) occurred before that of the GFP-positive dendritic branch (green; solid arrowhead). The original position of the dendritic tip is indicated by a dashed line. Scale bar, $5 \mu \mathrm{m}$. B, Neurons were infected with AAV9 GFP virus or AAV9 E6AP virus for $10 \mathrm{~d}$, and cleaved tubulin levels were measured by Western blot with an antibody specifically against the cleaved microtubule ( $\Delta$ Tubulin). C, Quantification showed an increased level of microtubule cleavage in E6AP-infected neurons; $n=3$ independent experiments. $\boldsymbol{D}$, Representative image of E6AP neurons immunostained with $\Delta$ Tubulin. Scale bar, $10 \mu \mathrm{m}$. $\boldsymbol{E}$, Quantification of $\Delta$ Tubulin immunointensity in neurons transfected with vector control, E6AP, or E6AP + Casp3 C163A (E6AP + (3 mutant), compared with control; $n=10$. F, Morphology of neurons transfected with surfGFP, tubulin WT, E6AP, or E6AP + tubulin WT. Scale bar, $50 \mu \mathrm{m}$. G, Sholl analysis of dendritic arborization; $n=10$ cells per condition. $\boldsymbol{H}$, Quantification of total dendritic length; $n=10$ cells per condition. Changes in tubulin stabilization also affected the E6AP-dependent dendritic remodeling (Fig. 5-1, available at https://doi.org/10.1523/JNEUROSCI.1511-17.2017.f5-1. Error bars represent SEM, ${ }^{*} p<0.05,{ }^{* *} p<0.01,{ }^{* * *} p<$ $0.001,{ }^{* * * *} p<0.0001$.

microtubule ahead of the retreating branch tip indicated by surfGFP (Fig. 5A).

Given that microtubules are the core supporting structure in dendrites, it is conceivable that activation of the caspase cascade triggers microtubule destruction and structural removal. Indeed, the cleavage of tubulin by caspase- 3 and caspase- 6 has been shown to be involved in cytoskeletal degradation during axon degeneration (Sokolowski et al., 2014). We therefore wondered 
whether tubulin cleavage occurs in dendrite remodeling. In neurons infected with GFP or E6AP AAV9 virus for $10 \mathrm{~d}$, we examined levels of cleaved tubulin by Western blot with the Tub $\Delta$ Casp6 antibody, which specifically recognizes the tubulin sites cleaved by caspase- 6 and caspase- 3 (Klaiman et al., 2008; Sokolowski et al., 2014). Surprisingly, in neurons infected with E6AP virus, increased levels of cleaved tubulin were detected (E6AP, $1.2 \pm 0.02, n=3, p=0.0006$; Fig. $5 B, C$ ). To further characterize this cleavage, we immunostained with the Tub $\Delta$ Casp 6 antibody in E6AP-transfected neurons. Compared with the control, much higher levels of cleaved tubulin were detected in E6APtransfected cells $\left(F_{(2,111)}=5.27, p=0.0065\right.$, ANOVA; Fig. $\left.5 D, E\right)$. Upon characterization of the localization of the cleaved tubulin signals, we found increased cleaved tubulin, especially in minor dendrites, which are those mostly affected by E6AP-induced remodeling (E6AP, $1.7 \pm 0.2, n=10, p<0.0001$; Fig. $5 D, E$ ). Interestingly, in cells cotransfected with E6AP and the caspase-3 mutant C163A, immunostaining signals of cleaved tubulin were dramatically reduced ( $1.2 \pm 0.1$ over control, $n=10, p>0.05$; Fig. $5 E$ ), indicating that the E6AP-dependent tubulin cleavage was dependent on caspase-3 activity.

To further assess the role of tubulin in E6AP-induced dendritic remodeling, we overexpressed E6AP along with tubulin. In the presence of higher levels of tubulin expression, E6APexpressing neurons no longer underwent morphological changes (Fig. $5 F-H$ ). Interestingly, when we overexpressed a less stable form of tubulin, the acetylation mutant tubulin K40A, along with E6AP, the increase in K40A tubulin failed to block the E6APinduced reduction in dendritic arborization (Figs. 5H, 5-1 A, $B$, available at https://doi.org/10.1523/JNEUROSCI.1511-17.2017. f5-1). These findings indicate that the integrity of tubulin or microtubules serves as a key substrate in E6AP-dependent dendritic remodeling. To further examine this idea, we treated control and E6AP-expressing neurons with $5 \mathrm{~nm}$ taxol to stabilize tubulin. Indeed, we found that treatment with taxol prevented E6APinduced morphological changes (Fig. $5 H, 5-1 C, D$, available at https://doi.org/10.1523/JNEUROSCI.1511-17.2017.f5-1). These results support the role of tubulin stability as an important determinant in dendritic remodeling caused by E6AP.

\section{E6AP-overexpression ASD mouse model shows normal neuronal density and cortical-layer formation}

Abnormal overexpression of E6AP in the brain is directly linked to the pathogenesis of autism. We therefore wanted to know whether similar cellular regulation and molecular cascades occur in vivo. We thus obtained the recently established E6AP autism mouse model. The $2 \mathrm{X}$ Tg transgenic mouse model exhibits a tripling of the normal Ube3A gene dosage in neurons, replicating idic15 in patients with autism (Smith et al., 2011). The increased levels of E6AP in these mice leads to a recapitulation of the three core behavioral autism traits: defective social interaction, impaired communication, and increased repetitive behavior. In addition, recordings in hippocampal slices showed reduced strength in synaptic transmission (Smith et al., 2011).

We first examined the developmental time course of E6AP expression in both WT and 2X Tg animals. Hippocampal brain samples were collected from P5-P40 mice and subjected to Western blotting to measure E6AP protein levels. In WT mice, E6AP was expressed at a peak level at P5 and P10, which then started declining at P15 until reaching minimal traces at P40. As expected, 2X Tg mice showed significantly higher levels of E6AP during the examined developmental period (2x Tg: P5, $1.67 \pm$ 0.32 of WT control, $p=0.16 ; \mathrm{P} 10,1.48 \pm 0.36, p=0.041 ; \mathrm{P} 15$,
$1.07 \pm 0.29, p=0.15 ; \mathrm{P} 20,0.85 \pm 0.29, p=0.13 ; \mathrm{P} 40,0.35 \pm$ $0.17, p=0.015, n=3$ for all time points; Fig. $6 A, B)$. Interestingly, $2 \mathrm{X}$ Tg mice shared the same time course pattern of E6AP expression (Fig. $6 A, B$ ). To visualize the E6AP distribution pattern in the cortex, we then immunostained E6AP in brain slices of P15 mice. Consistent with Western blotting, E6AP immunointensity in $2 \mathrm{X}$ Tg cortical slices was significantly stronger in all cortical layers (2.5 \pm 0.3 of WT, $n=20$ slices, $p<0.0001$; Fig. $6 C, D)$.

To determine the effect of E6AP overexpression on overall brain development, P15 brain slices were labeled with Hoechst nuclear dye to indicate structural organization. Examination of the $2 \mathrm{X}$ Tg slices revealed normal cortical-layer patterns of layers I-VI, and the thickness of each cortical layer was similar to that of the WT animals (Fig. 6-1A,B, available at https://doi.org/10. 1523/JNEUROSCI.1511-17.2017.f6-1). Next, we stained P15 slices for the neuron-specific marker NeuN and found similar distribution and cell density of neurons within the cortex (Fig. 6-1C,D, available at https://doi.org/10.1523/JNEUROSCI. 1511-17. 2017.f6-1). To further examine the effect of high E6AP levels on cellular organization, we infected brains at P0 with AAV2 GFP virus by intraventricular injection, and brain slices were collected at P40 to allow sufficient GFP intensity. Infected cortical pyramidal neurons showed regular distribution and normal gross cellular structure with the single primary dendrite projecting to the pia in both WT and $2 \mathrm{X} \mathrm{Tg}$ mice (Fig. 6E). These findings indicate that the increased dosage of E6AP did not cause significant impairments in overall neurogenesis and cortical structural development.

\section{XIAP, caspase-3, and tubulin cleavage are involved in the E6AP ASD mouse model brain}

In primary cultured neurons, we have shown that E6AP targeted XIAP for ubiquitination and degradation. We wondered whether XIAP was also downregulated in this transgenic ASD mouse model. We collected hippocampal brain tissue from mice at P15 and examined XIAP protein levels. Indeed, we observed a significant decrease in XIAP levels in $2 \mathrm{X}$ Tg mice compared with WT control $(0.51 \pm 0.07$ of the control, $n=3, p=0.042$; Fig. $7 A, B)$. We also stained slices for XIAP to determine the pattern of XIAP decrease among different cortical layers. We found that in slices obtained from $2 \mathrm{X}$ Tg mice, XIAP immunointensity decreased in all the cortical layers, with the overall intensity reduced to $0.48 \pm$ 0.03 of the WT control $(n=20$ slices, $p<0.0001$; Fig. $6 F, G)$. Given the elevated amount of E6AP in $2 \mathrm{X}$ Tg mice, we assumed that changes in XIAP resulted from enhancement in protein ubiquitination. To determine the general ubiquitination levels in the transgenic mouse brain and prevent rapid protein degradation, we tried to accumulate ubiquitinated proteins in the mouse brains by injecting the proteasomal inhibitor MG132 (10 mM, 1.5 $\mu \mathrm{l}$ in each ventricle) into both ventricles (Villamar-Cruz et al., 2006; Wójcik et al., 2015). Hippocampal and cortical brain tissues were collected $12 \mathrm{~h}$ later for Western analysis. Indeed, in the MG132-treated brain lysates, ubiquitination signals were increased in $2 \mathrm{X}$ Tg samples (Fig. $6 H$ ).

We have shown in cultured neurons that XIAP reduction led to activation of the caspase cascade. We wondered whether the same signaling occurred in the brain of the E6AP-overexpressing ASD mouse model. We collected hippocampal brain-tissue samples from mice at P15 and measured the cleaved form (i.e., active form) of caspase-3 by Western blotting. Compared with WT mice, cleaved caspase-3 levels were significantly increased in $2 \mathrm{X}$ Tg mice ( $1.4 \pm 0.01$-fold increase, $n=3, p=0.0097$; Fig. $7 A, C$ ), paired with a decrease in caspase- 3 levels (Fig. $7 A$ ). Consistent 
A
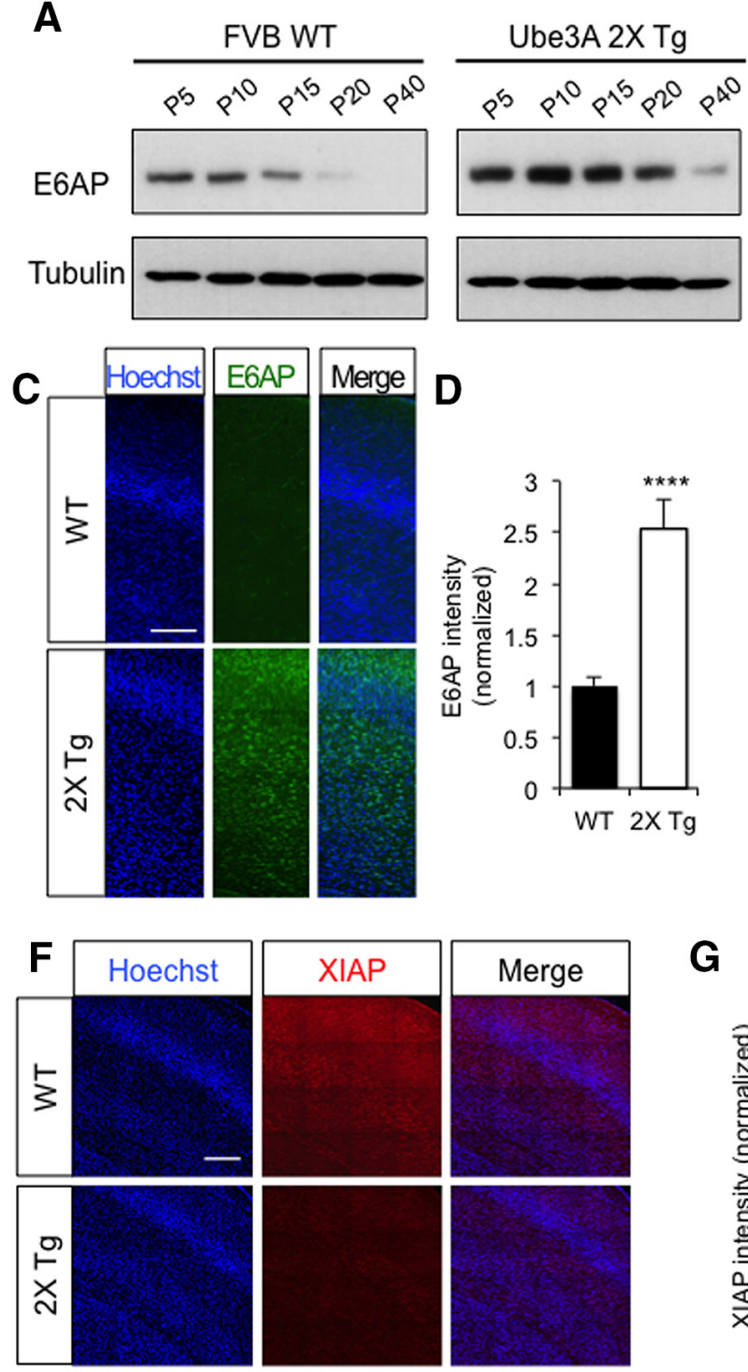
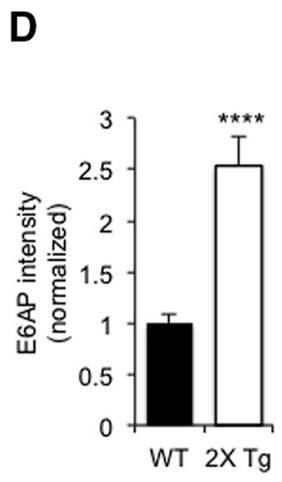

B

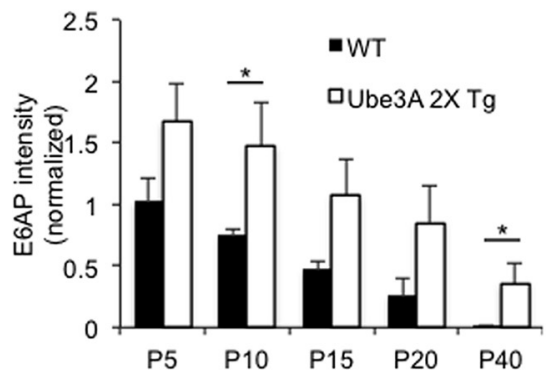

E
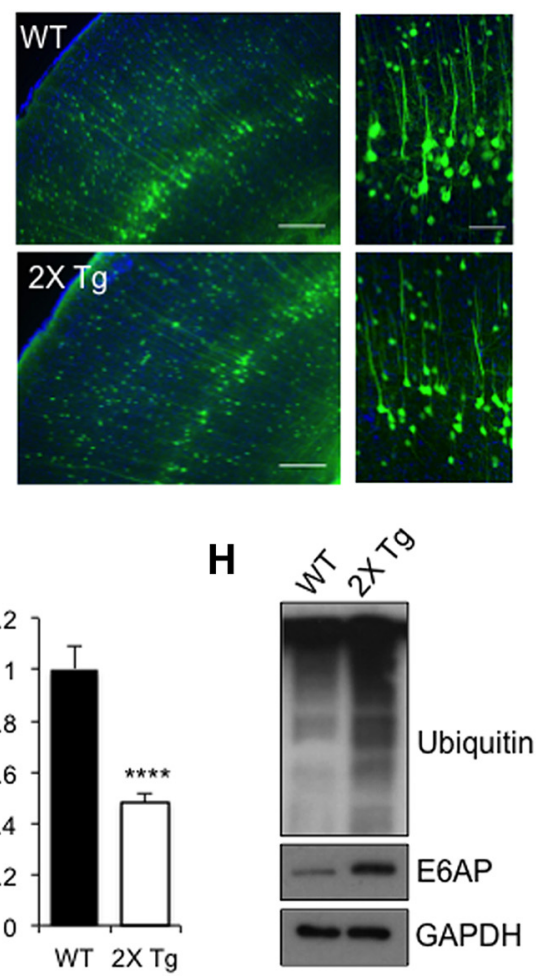

Figure 6. Increased E6AP overexpression in 2X Tg mice leads to decreased XIAP expression. A, Hippocampal brain lysates were collected from WT or 2 X Tg mice from P5 to P40 and E6AP levels were measured by Western blot. Tubulin was probed as a loading control. $B$, Quantification of E6AP Western blot intensity; $n=3$ independent experiments. $C$, Immunostaining of E6AP in somatosensory cortex slices obtained from P15 WT or 2X Tg mice. Scale bar, $100 \mu \mathrm{m}$. D, Quantification showed an increase in E6AP signal intensity in Tg mice; $n=20$ slices. $E$, GFP AAV2 virus was injected into the brain ventricles of WT and $2 X \mathrm{Xg}$ mice at PO. Brain slices were prepared at P40 and imaged. Scale bar, $100 \mu \mathrm{m}$. A portion of the image of layer-V neurons was enlarged for clarity. Scale bar, $50 \mu \mathrm{m}$. Cortical-layer development and neuron density were also analyzed in 2X Tg mice (Fig. 6-1, available at https://doi.org/10.1523/JNEUROSCI.1511-17.2017.f6-1). $F$, XIAP staining in somatosensory cortex slices from P15 WT or $2 X$ Tg mice. Scale bar, $100 \mu \mathrm{m}$. G, Quantification showed a decrease in XIAP signal intensity in $2 X$ Tg mice; $n=20$ slices. $\boldsymbol{H}, \mathrm{MG} 132$ (10 mM, $1.5 \mu$ lin each ventricle) was injected into the brain of both WT and $2 X \mathrm{Tg}$ mice at P3 for $12 \mathrm{~h}$. Brain lysates were probed for ubiquitin signals. An elevated ubiquitination amount was detected in $2 \mathrm{X}$ Tg mice under MG132 treatment. Error bars represent SEM, ${ }^{*} p<0.05,{ }^{* * * *} p<0.0001$.

with our findings in cultured neurons, we observed that cleaved tubulin levels were also increased in the $2 \mathrm{X}$ Tg animals (1.4 \pm 0.04 times that of the WT control, $n=3, p=0.0089$; Fig. $7 A, D)$.

E6AP ASD mouse model neurons show impairment in spine maturation and reduction in dendritic branching

In the E6AP transgenic ASD mouse model, we detected molecular and signaling regulation similar to that found in E6APtransfected neurons. We therefore wanted to determine whether these molecular changes were accompanied by morphological alteration in neurons. Brain slices were prepared from the somatosensory cortex of P15 mouse brains after Golgi staining, and the spines at the basolateral dendrites were measured in layer- $\mathrm{V}$ pyramidal neurons. Representative spine images and spine tracings are shown in Figure $7 E$. Similar to its effects in cultured neurons, increased E6AP levels in the mouse brain led to a decrease in spine density (WT, $1.7 \pm 0.1$ spines/ $\mu \mathrm{m} ; 2 \mathrm{X}$ Tg, $1.4 \pm$
0.1 spines $/ \mu \mathrm{m}, n=10$ neurons, $p=0.019$; Fig. $7 E, F)$. Although spine density decreased, mean spine length increased in $2 \mathrm{X} \mathrm{Tg}$ neurons (WT, $1.2 \pm 0.3 \mu \mathrm{m} /$ spine; $2 \mathrm{X} \mathrm{Tg}, 1.3 \pm 0.3 \mu \mathrm{m} / \mathrm{spine}$, $n=10, p=0.007$; Fig. $7 G$ ). Since we saw an increase in filopodia in culture neurons, we wondered whether the increase in spine length suggests a similar change in transgenic animal spines. Indeed, both the number and percentage of filopodia increased in $2 \mathrm{X}$ Tg mice (for filopodia number: WT, $1.6 \pm 0.4$ filopodia/50 $\mu \mathrm{m}$; $2 \mathrm{X} \mathrm{Tg}, 3.2 \pm 0.7$ filopodia/50 $\mu \mathrm{m}, n=10, p=0.03$; for filopodia percentage: WT, $1.8 \pm 1.1 \% ; 2 \mathrm{X} \mathrm{Tg}, 4.4 \pm 1.4 \%, n=10$, $p=0.04$; Fig. $7 \mathrm{H}, \mathrm{I})$. These results suggest that in $2 \mathrm{X} \mathrm{Tg}$ mice, an increase in E6AP levels resulted in suppression of spine formation and/or maturation, leading to a decrease in spine density and an increase in filopodia.

As increased E6AP levels in cultured neurons lead to a reduction in dendritic arborization by dendritic retraction, we wanted to determine whether this also occurred in the neurons of $2 \mathrm{X} \mathrm{Tg}$ 

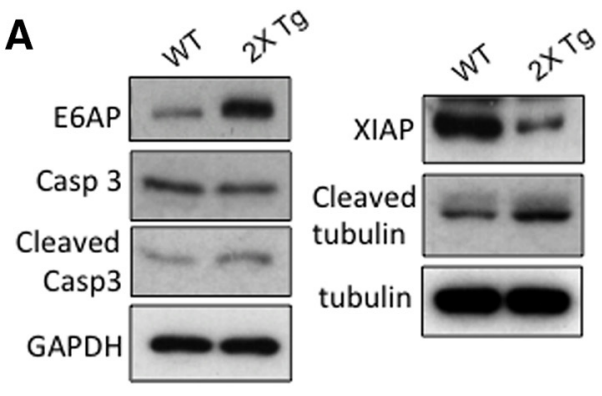

E
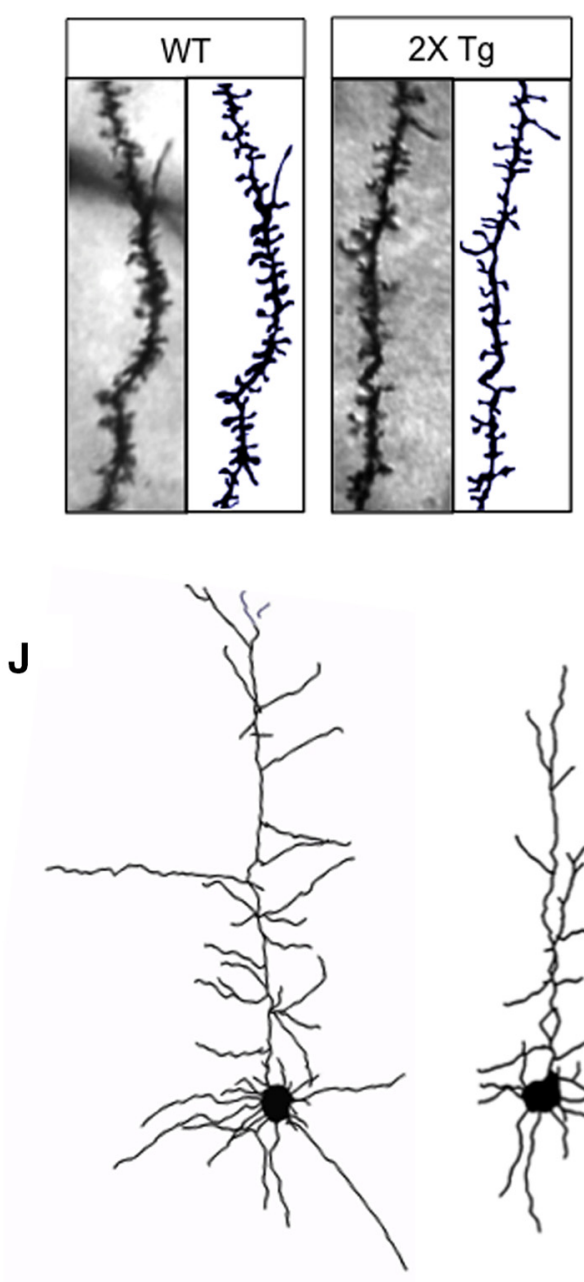

WT

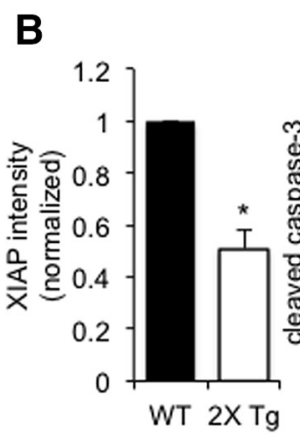

C

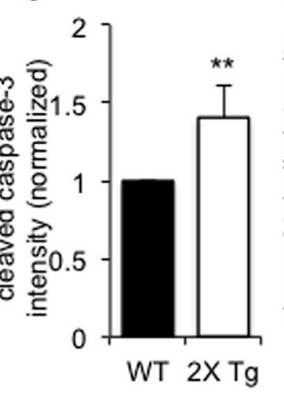

F

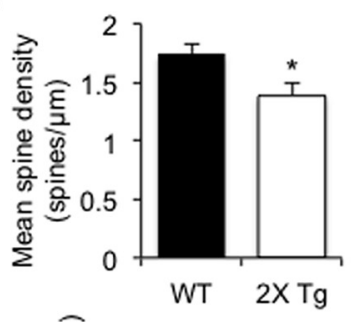

H

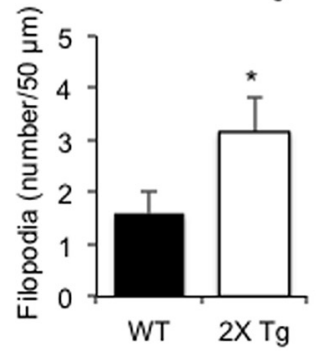

K

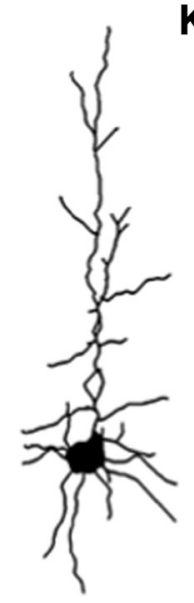

$2 \times \mathrm{Tg}$
G

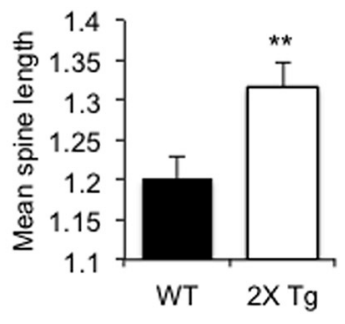

I

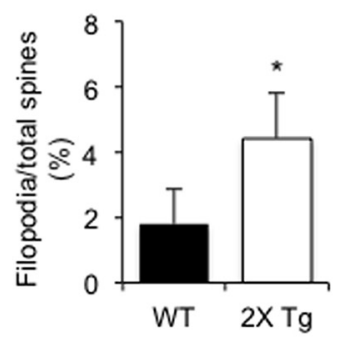

L

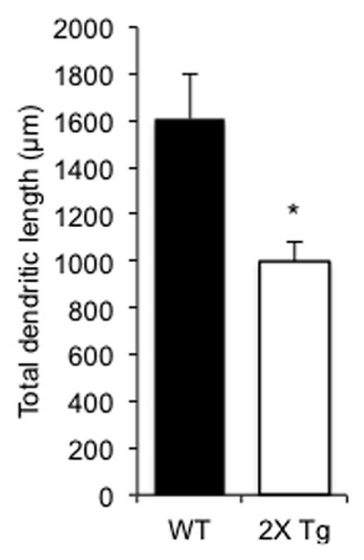

Figure 7. E6AP autism mouse model neurons show impairment in spine maturation and reduction in dendritic branching. $A$, Brain lysates collected from WT or $2 X \mathrm{Xg}$ mice at P15 were probed for E6AP, XIAP, caspase-3, cleaved caspase-3, cleaved tubulin, and total tubulin. GAPDH was also probed as a loading control. $\boldsymbol{B}-\boldsymbol{D}$, Quantification analysis of Western blots for XIAP, cleaved caspase-3, and cleaved tubulin; $n=3$ for each. E, At P15, brains of WT and $2 X$ Tg mice were subjected to Golgi staining. Representative images of spine morphology of layer-V somatosensory cortical neurons are shown. $\boldsymbol{F}$, Mean spine density decreased in $2 X \operatorname{Tg}$ mice; $n=10$ neurons. $\boldsymbol{G}$, Mean spine length increased in $2 X \operatorname{Tg}$ mice; $n=10$ neurons. $\boldsymbol{H}, \boldsymbol{I}$, The percentage and number of filopodia increased in $2 X \operatorname{Tg}$ mice; $n=10$ neurons. $J$, Representative layer-V pyramidal neuron tracing images of Golgi staining from P15 WT and 2X Tg mouse brain slices. $K, L$, Measurement of average dendrite number and total dendritic length in pyramidal neurons; $n=12$ neurons. Error bars represent SEM, ${ }^{*} p<0.05,{ }^{* *} p<0.01$ (Fig. 7-1, available at https://doi.org/10.1523/JNEUROSCl.1511-17.2017.f7-1). Summary of the E6AP-dependent dendritic remodeling pathway.

animals. We subsequently collected brain slices at P15 and subjected them to Golgi staining to study morphology of layer-V pyramidal neurons in the somatosensory cortex. Compared with WT animals, the mean number of dendritic branches per cell was significantly decreased in $2 \mathrm{X}$ Tg animals (WT, $31 \pm 2.9$ dendrites, $n=13 ; 2 \mathrm{X} \mathrm{Tg}, 18.6 \pm 1.4$ dendrites, $n=12, p=0.033 ;$ Fig. $7 J, K$ ). Along with fewer dendrites, total dendritic length in neurons was also markedly reduced in $2 \mathrm{X}$ Tg mice (WT, $1608 \pm 187 \mu \mathrm{m}, n=$ 13; $2 \mathrm{X} \mathrm{Tg}, 998 \pm 85 \mu \mathrm{m}, n=12, p=0.013$; Fig. $7 L$ ). These results suggest a defect in dendritic development in $2 \mathrm{X} \mathrm{Tg}$ mice as a 
result of increased E6AP expression in the brain. These in vivo findings support the idea that E6AP has a role in downregulation of dendritic arborization, presumably by dendritic retraction mediated by the molecular pathway involving the E6APinduced ubiquitination and degradation of XIAP, the subsequent activation of caspase-3, and the resulting cleavage of tubulin and local dendritic degeneration (Fig. 7-1, available at https://doi.org/10.1523/JNEUROSCI.1511-17.2017.f7-1).

\section{Discussion}

In this study we elucidate for the first time the molecular mechanisms underlying Ube3A/E6AP-dependent regulation of dendritic arborization. We show, both in vitro in cultured neurons and in vivo in the E6AP ASD mouse model, that increased levels of E6AP causes XIAP ubiquitination and degradation, resulting in activation of the caspase cascade, which ultimately leads to reduced dendritic arborization via tubulin cleavage and dendritic retraction. These neurodevelopmental deficits and the underlying mechanistic cascades represent the molecular pathology of ASD resulting from aberrant upregulation of E6AP expression.

Sholl analysis of different DIV time points showed that the reduction in arborization was not due to a suppression of growth, indicating active dendrite retraction. Consistently, live-imaging experiments revealed the mechanism of dendritic remodeling in E6AP-overexpressing neurons. We observed distal fragmentation and thinning of dendritic branches, followed by tip retraction and eventual disappearance of dendrites. In line with our finding on the role of E6AP in dendritic remodeling, knockdown of E6AP in mouse pyramidal neurons showed disrupted apical dendrites, which is also observed in the maternally deficient Ube3A AS mouse model (Miao et al., 2013). In addition, a study of Drosophila dendritic arborization neurons has shown that loss of the E6AP homolog $d U B E 3 A$ alters terminal dendritic branching and growth (Lu et al., 2009).

We identify XIAP as a novel ubiquitination and degradation target for E6AP E3 ligase activity. The role of XIAP has previously been implicated in axon degeneration. In the absence of XIAP, dorsal root ganglion (DRG) axons subjected to nerve growth factor withdrawal show accelerated degeneration and increased caspase- 3 activity, along with decreased levels of XIAP (Unsain et al., 2013). Furthermore, sustaining XIAP levels in degenerating axons reduces caspase activation and suppresses axonal degeneration (Unsain et al., 2013). Consistent with these studies, we find that XIAP levels are decreased during dendritic remodeling, and that the reinstatement of XIAP rescues E6AP-dependent structural reorganization.

There are two possible mechanisms by which XIAP could regulate caspase activation in E6AP-dependent dendritic remodeling. First, as XIAP inhibits both the cleavage of caspase- 3 and its access to target substrates (Chai et al., 2001; Riedl et al., 2001), lowering XIAP levels by E6AP-mediated ubiquitination and degradation removes XIAP's inhibition on caspases. This would allow caspases to be activated and act on their substrates. Second, XIAP can directly target caspase- 3 for ubiquitination and proteasomal degradation (Suzuki et al., 2001; Schile et al., 2008). Our immunostaining and Western data demonstrate that E6APinduced XIAP reduction is accompanied with an increase in caspase- 3 cleavage and thus activation, indicating an alleviation of XIAP inhibition in the presence of higher levels of E6AP.

We found caspases to be key components in the E6APdependent dendritic remodeling pathway. Caspases have been shown to play a role in axon pruning of NGF-dependent DRGs (Nikolaev et al., 2009; Simon et al., 2012; Cusack et al., 2013). In cultured hippocampal neurons, local activation of caspase-3 by Mito-KillerRed photostimulation was sufficient to induce proteasome-dependent spine elimination and dendrite retraction without cell death (Ertürk et al., 2014). In agreement with these studies, we demonstrate that the E6AP-induced remodeling process in neuronal cultures is dependent on caspase- 3 cleavage and activity indicated by pharmacological inhibition and rescue experiments. However, we observed that the distribution of activated caspase- 3 is not limited to the dendritic arbors or individual branches; rather, strong signals were also detected in the soma. Global caspase activation has been shown to cause widespread damage leading to apoptosis (Yuan and Yankner, 2000). However, we did not observe cell death even $7 \mathrm{~d}$ after E6AP transfection (data not shown). In addition, in $2 \mathrm{X}$ Tg mice with tripled E6AP dosage and increased levels in cleaved caspase-3, Hoechst and NeuN staining in cortical slices failed to show any changes in neuron number, indicating a lack of neuron apoptosis in the presence of E6AP-induced caspase activation. We therefore wondered whether E6AP overexpression also triggers an upregulation of prosurvival signaling cascade(s), such as the PI3K-Akt pathway, protecting neurons from overall degeneration. Indeed, in neurons transfected with E6AP, we detected an upregulation of phosphorylated and activated Akt (data not shown). Consistently, increased p-Akt levels were also detected in neurons infected with AAV9 E6AP virus and in 2X Tg mouse brain tissue (data not shown).

We observed retraction of tubulin from the tip of the dendrite, which preceded retraction of the neuronal structure itself. However, cleavage of tubulin by caspases was observed on the dendrite at locations more proximal to the cell body rather than at the tip, suggesting a link between proximal cleavage of tubulin and distal degeneration. It is possible that microtubule cleavage blocks the trafficking of molecular cargo to reach the distal ends, leading to degeneration and retraction of the distal dendritic fragment. These findings provide insight into the molecular mechanism of E6AP-dependent dendritic remodeling.

Importantly, our in vitro findings were validated in the Ube3A-overexpressing ASD mouse model. The $2 \mathrm{X}$ Tg mice show typical ASD behavioral deficits, including impaired social behavior (as measured by social-preference tests), decreased communication (measured by vocalizations), and increased repetitive behavior (shown by excessive grooming; Smith et al., 2011). In these transgenic animals, we measured neuron number and cortical-layer structure and thickness and found no obvious changes. In postnatal cortical neurons, the structural pattern and orientation of the apical dendrites appeared normal. These findings indicate relatively normal brain development, including neurogenesis and neuron migration. In contrast, detailed analysis revealed alterations in spine formation and dendritic branching. Reduced spine density and increased immature filopodia-like spines in $2 \mathrm{X}$ Tg neurons suggests suppression in spinogenesis, stability, or maturation. These findings are consistent with the electrophysiological changes we found in hippocampal neurons, as well as a previously reported decrease of mEPSC frequency in 2X Tg mice (Smith et al., 2011). Consistent with our in vitro studies, $2 \mathrm{X}$ Tg mice showed a reduction in dendritic branching in layer-V cortical neurons. Strikingly, the same signaling cascades for dendritic remodeling observed in E6AP-transfected neurons were also used in $2 \mathrm{X}$ Tg mice. The animal brains showed decreased XIAP levels, increased caspase- 3 cleavage, and enhanced tubulin cleavage, supporting the involvement of these key components in E6AP-mediated morphological remodeling.

We show that E6AP is expressed in the brain mainly during early development, and is then reduced to and maintained at a 
minimal level, which is consistent with the fact that E6AP mRNA peaks in the mouse brain at a critical period in development (Kroon et al., 2013). During this critical window, neuronal dendrites are remodeled in a developmental-dependent and activitydependent manner to form more specific stable connections as neurons mature (Koleske, 2013). In the visual cortex of AS mice, E6AP has been shown to regulate experience-dependent neuronal development from P10 to P25 (Yashiro et al., 2009; Kim et al., 2016). Thus, it is likely that E6AP is involved in dendritic remodeling during the critical window of neuron dendrite development and maturation. Indeed, in an AS model with maternal E6AP deficiency, reinstatement of E6AP expression at birth and at 3 weeks of age was able to rescue motor deficits, while reinstatement in adults failed to show rescue effects (Silva-Santos et al., 2015). As we observed a shared time course in E6AP expression in both WT and $2 \mathrm{X}$ Tg mice, we predict that with the overexpression of E6AP, the overall time course of developmental dendritic remodeling may remain the same, but the dendritic arbor is pathologically overpruned due to elevated E6AP activity. Future rescue studies on the E6AP ASD mouse model will confirm the existence of a critical window, which will provide valuable guidance on clinical therapeutics of ASD patients.

Aberrant connectivity and thus malfunction of neural circuitry is one of the major common developmental changes in ASD (Ebert and Greenberg, 2013; Doll and Broadie, 2014). Our findings on ASD-related alterations in dendritic remodeling and spine formation during brain development provide mechanistic insights at the cellular and molecular levels. In E6AP-expressing neurons and in the brain of the E6AP ASD mouse model, we show alterations in spine density and maturation. Consistently, electrophysiological recordings revealed changes in synaptic activity. Furthermore, reduced dendritic arborization is expected to cause a decrease in the complexity of neuron connectivity. These cellular developmental defects may result in improper wiring of neuronal circuitry, and therefore contribute to the development of altered social and cognitive behaviors in ASDs.

\section{References}

Albrecht U, Sutcliffe JS, Cattanach BM, Beechey CV, Armstrong D, Eichele G, Beaudet AL (1997) Imprinted expression of the murine Angelman syndrome gene, Ube3a, in hippocampal and Purkinje neurons. Nat Genet 17:75-78. CrossRef Medline

Berman RF, Murray KD, Arque G, Hunsaker MR, Wenzel HJ (2012) Abnormal dendrite and spine morphology in primary visual cortex in the CGG knock-in mouse model of the fragile X premutation. Epilepsia 53 [Suppl 1]:150-160. CrossRef Medline

Campbell DS, Okamoto H (2013) Local caspase activation interacts with Slit-Robo signaling to restrict axonal arborization. J Cell Biol 203:657672. CrossRef Medline

Chai J, Shiozaki E, Srinivasula SM, Wu Q, Datta P, Alnemri ES, Shi Y, Dataa P (2001) Structural basis of caspase-7 inhibition by XIAP. Cell 104:769780. CrossRef Medline

Cline HT (2001) Dendritic arbor development and synaptogenesis. Curr Opin Neurobiol 11:118-126. CrossRef Medline

Crinelli R, Bianchi M, Menotta M, Carloni E, Giacomini E, Pennati M, Magnani M (2008) Ubiquitin over-expression promotes E6AP autodegradation and reactivation of the p53/MDM2 pathway in HeLa cells. Mol Cell Biochem 318:129-145. CrossRef Medline

Cusack CL, Swahari V, Hampton Henley W, Michael Ramsey J, Deshmukh M (2013) Distinct pathways mediate axon degeneration during apoptosis and axon-specific pruning. Nat Commun 4:1876. CrossRef Medline

de Anda FC, Rosario AL, Durak O, Tran T, Gräff J, Meletis K, Rei D, Soda T, Madabhushi R, Ginty DD, Kolodkin AL, Tsai LH (2012) Autism spectrum disorder susceptibility gene TAOK2 affects basal dendrite formation in the neocortex. Nat Neurosci 15:1022-1031. CrossRef Medline

Deveraux QL, Takahashi R, Salvesen GS, Reed JC (1997) X-linked IAP is a direct inhibitor of cell-death proteases. Nature 388:300-304. CrossRef Medline

Doll CA, Broadie K (2014) Impaired activity-dependent neural circuit assembly and refinement in autism spectrum disorder genetic models. Front Cell Neurosci 8:30. CrossRef Medline

Ebert DH, Greenberg ME (2013) Activity-dependent neuronal signalling and autism spectrum disorder. Nature 493:327-337. CrossRef Medline

Ertürk A, Wang Y, Sheng M (2014) Local pruning of dendrites and spines by caspase-3-dependent and proteasome-limited mechanisms. J Neurosci 34:1672-1688. CrossRef Medline

Hogart A, Wu D, LaSalle JM, Schanen NC (2010) The comorbidity of autism with the genomic disorders of chromosome 15q11.2-q13. Neurobiol Dis 38:181-191. CrossRef Medline

Hou Q, Huang Y, Amato S, Snyder SH, Huganir RL, Man HY (2008) Regulation of AMPA receptor localization in lipid rafts. Mol Cell Neurosci 38:213-223. CrossRef Medline

Huo Y, Khatri N, Hou Q, Gilbert J, Wang G, Man HY (2015) The deubiquitinating enzyme USP46 regulates AMPA receptor ubiquitination and trafficking. J Neurochem 134:1067-1080. CrossRef Medline

Irwin SA, Patel B, Idupulapati M, Harris JB, Crisostomo RA, Larsen BP, Kooy F, Willems PJ, Cras P, Kozlowski PB, Swain RA, Weiler IJ, Greenough WT (2001) Abnormal dendritic spine characteristics in the temporal and visual cortices of patients with fragile-X syndrome: a quantitative examination. Am J Med Genet 98:161-167. CrossRef Medline

Jiao S, Li Z (2011) Nonapoptotic function of BAD and BAX in long-term depression of synaptic transmission. Neuron 70:758-772. CrossRef Medline

Kameda H, Furuta T, Matsuda W, Ohira K, Nakamura K, Hioki H, Kaneko T (2008) Targeting green fluorescent protein to dendritic membrane in central neurons. Neurosci Res 61:79-91. CrossRef Medline

Kim HC, Huibregtse JM (2009) Polyubiquitination by HECT E3s and the determinants of chain type specificity. Mol Cell Biol 29:3307-3318. CrossRef Medline

Kim H, Kunz PA, Mooney R, Philpot BD, Smith SL (2016) Maternal loss of Ube3a impairs experience-driven dendritic spine maintenance in the developing visual cortex. J Neurosci 36:4888-4894. CrossRef Medline

Klaiman G, Petzke TL, Hammond J, Leblanc AC (2008) Targets of caspase-6 activity in human neurons and Alzheimer disease. Mol Cell Proteomics 7:1541-1555. CrossRef Medline

Koester SE, O'Leary DD (1992) Functional classes of cortical projection neurons develop dendritic distinctions by class-specific sculpting of an early common pattern. J Neurosci 12:1382-1393. Medline

Koleske AJ (2013) Molecular mechanisms of dendrite stability. Nat Rev Neurosci 14:536-550. CrossRef Medline

Kozlowski DA, Schallert T (1998) Relationship between dendritic pruning and behavioral recovery following sensorimotor cortex lesions. Behav Brain Res 97:89-98. CrossRef Medline

Kroon T, Sierksma MC, Meredith RM (2013) Investigating mechanisms underlying neurodevelopmental phenotypes of autistic and intellectual disability disorders: a perspective. Front Syst Neurosci 7:75. CrossRef Medline

Levy SE, Mandell DS, Schultz RT (2009) Autism. Lancet 374:1627-1638. CrossRef Medline

Li Z, Jo J, Jia JM, Lo SC, Whitcomb DJ, Jiao S, Cho K, Sheng M (2010) Caspase- 3 activation via mitochondria is required for long-term depression and AMPA receptor internalization. Cell 141:859-871. CrossRef Medline

Lin A, Hou Q, Jarzylo L, Amato S, Gilbert J, Shang F, Man HY (2011) Nedd4-mediated AMPA receptor ubiquitination regulates receptor turnover and trafficking. J Neurochem 119:27-39. CrossRef Medline

Lu Y, Wang F, Li Y, Ferris J, Lee JA, Gao FB (2009) The Drosophila homologue of the Angelman syndrome ubiquitin ligase regulates the formation of terminal dendritic branches. Hum Mol Genet 18:454-462. CrossRef Medline

Man HY, Sekine-Aizawa Y, Huganir RL (2007) Regulation of \{alpha\}amino-3-hydroxy-5-methyl-4-isoxazolepropionic acid receptor trafficking through PKA phosphorylation of the Glu receptor 1 subunit. Proc Natl Acad Sci U S A 104:3579-3584. CrossRef Medline

McGee A, Li G, Lu Z, Qiu S (2014) Convergent synaptic and circuit substrates underlying autism genetic risks. Front Biol (Beijing) 9:137-150. CrossRef Medline

Miao S, Chen R, Ye J, Tan GH, Li S, Zhang J, Jiang YH, Xiong ZQ (2013) The Angelman syndrome protein Ube3a is required for polarized dendrite 
morphogenesis in pyramidal neurons. J Neurosci 33:327-333. CrossRef Medline

Mocanu MM, Baxter GF, Yellon DM (2000) Caspase inhibition and limitation of myocardial infarct size: protection against lethal reperfusion injury. Br J Pharmacol 130:197-200. CrossRef Medline

Mukaetova-Ladinska EB, Arnold H, Jaros E, Perry R, Perry E (2004) Depletion of MAP2 expression and laminar cytoarchitectonic changes in dorsolateral prefrontal cortex in adult autistic individuals. Neuropathol Appl Neurobiol 30:615-623. CrossRef Medline

Nikolaev A, McLaughlin T, O’Leary DD, Tessier-Lavigne M (2009) APP binds DR6 to trigger axon pruning and neuron death via distinct caspases. Nature 457:981-989. CrossRef Medline

Parrish JZ, Emoto K, Kim MD, Jan YN (2007) Mechanisms that regulate establishment, maintenance, and remodeling of dendritic fields. Annu Rev Neurosci 30:399-423. CrossRef Medline

Pathania M, Davenport EC, Muir J, Sheehan DF, López-DoménechG, Kittler JT (2014) The autism and schizophrenia associated gene CYFIP1 is critical for the maintenance of dendritic complexity and the stabilization of mature spines. Transl Psychiatry 4:e374. CrossRef Medline

Puram SV, Kim AH, Bonni A (2010) An old dog learns new tricks: a novel function for Cdc20-APC in dendrite morphogenesis in neurons. Cell Cycle 9:482-485. CrossRef Medline

Rajcan-Separovic E, Liston P, Lefebvre C, Korneluk RG (1996) Assignment of human inhibitor of apoptosis protein (IAP) genes xiap, hiap-1, and hiap-2 to chromosomes Xq25 and 11q22-q23 by fluorescence in situ hybridization. Genomics 37:404-406. CrossRef Medline

Raymond GV, Bauman ML, Kemper TL (1996) Hippocampus in autism: a Golgi analysis. Acta Neuropathol 91:117-119. Medline

Riedl SJ, Renatus M, Schwarzenbacher R, Zhou Q, Sun C, Fesik SW, Liddington RC, Salvesen GS (2001) Structural basis for the inhibition of caspase- 3 by XIAP. Cell 104:791-800. CrossRef Medline

Roy N, Deveraux QL, Takahashi R, Salvesen GS, Reed JC (1997) The c-IAP-1 and c-IAP-2 proteins are direct inhibitors of specific caspases. EMBO J 16:6914-6925. CrossRef Medline

Schile AJ, García-FernándezM, Steller H (2008) Regulation of apoptosis by XIAP ubiquitin-ligase activity. Genes Dev 22:2256-2266. CrossRef Medline

Silva-Santos S, van Woerden GM, Bruinsma CF, Mientjes E, Jolfaei MA, Distel B, Kushner SA, Elgersma Y (2015) Ube3a reinstatement identifies distinct developmental windows in a murine Angelman syndrome model. J Clin Invest 125:2069-2076. CrossRef Medline

Simon DJ, Weimer RM, McLaughlin T, Kallop D, Stanger K, Yang J, O’Leary DD, Hannoush RN, Tessier-Lavigne M (2012) A caspase cascade regulating developmental axon degeneration. J Neurosci 32:17540-17553. CrossRef Medline

Smith SE, Zhou YD, Zhang G, Jin Z, Stoppel DC, Anderson MP (2011) Increased gene dosage of Ube3a results in autism traits and decreased glutamate synaptic transmission in mice. Sci Transl Med 3:103ra97. CrossRef Medline

Sokolowski JD, Gamage KK, Heffron DS, Leblanc AC, Deppmann CD, Mandell JW (2014) Caspase-mediated cleavage of actin and tubulin is a com- mon feature and sensitive marker of axonal degeneration in neural development and injury. Acta Neuropathol Commun 2:16. CrossRef Medline

Suzuki Y, Nakabayashi Y, Nakata K, Reed JC, Takahashi R (2001) X-linked inhibitor of apoptosis protein (XIAP) inhibits caspase-3 and -7 in distinct modes. J Biol Chem 276:27058-27063. CrossRef Medline

Tao J, Rolls MM (2011) Dendrites have a rapid program of injury-induced degeneration that is molecularly distinct from developmental pruning. J Neurosci 31:5398-5405. CrossRef Medline

Unsain N, Barker PA (2015) New views on the misconstrued: executioner caspases and their diverse non-apoptotic roles. Neuron 88:461-474. CrossRef Medline

Unsain N, Higgins JM, Parker KN, Johnstone AD, Barker PA (2013) XIAP regulates caspase activity in degenerating axons. Cell Rep 4:751-763. CrossRef Medline

Verma P, Chierzi S, Codd AM, Campbell DS, Meyer RL, Holt CE, Fawcett JW (2005) Axonal protein synthesis and degradation are necessary for efficient growth cone regeneration. J Neurosci 25:331-342. CrossRef Medline

Villamar-Cruz O, Manjarrez-Marmolejo J, Alvarado R, Camacho-Arroyo I (2006) Regulation of the content of progesterone and estrogen receptors, and their cofactors SRC- 1 and SMRT by the $26 \mathrm{~S}$ proteasome in the rat brain during the estrous cycle. Brain Res Bull 69:276-281. CrossRef Medline

Williams CA, Beaudet AL, Clayton-Smith J, Knoll JH, Kyllerman M, Laan LA, Magenis RE, Moncla A, Schinzel AA, Summers JA, Wagstaff J (2006) Angelman syndrome 2005: updated consensus for diagnostic criteria. Am J Med Genet A 140:413-418. CrossRef Medline

Williams RS, Hauser SL, Purpura DP, DeLong GR, Swisher CN (1980) Autism and mental retardation: neuropathologic studies performed in four retarded persons with autistic behavior. Arch Neurol 37:749-753. CrossRef Medline

Wójcik S, Spodnik JH, Dziewiątkowski J, Spodnik E, Moryś J (2015) Morphological changes within the rat lateral ventricle after the administration of proteasome inhibitors. PLoS One 10:e0140536. CrossRef Medline

Yashiro K, Riday TT, Condon KH, Roberts AC, Bernardo DR, Prakash R, Weinberg RJ, Ehlers MD, Philpot BD (2009) Ube3a is required for experience-dependent maturation of the neocortex. Nat Neurosci 12: 777-783. CrossRef Medline

Young SS, Liston P, Xuan JY, McRoberts C, Lefebvre CA, Korneluk RG (1999) Genomic organization and physical map of the human inhibitors of apoptosis: HIAP1 and HIAP2. Mamm Genome 10:44-48. CrossRef Medline

Yuan J, Yankner BA (2000) Apoptosis in the nervous system. Nature 407: 802-809. CrossRef Medline

Zablotsky B, Black LI, Maenner MJ, Schieve LA, Blumberg SJ (2015) Estimated prevalence of autism and other developmental disabilities following questionnaire changes in the 2014 National Health Interview Survey. Natl Health Stat Report 1-20. Medline

Zehr JL, Todd BJ, Schulz KM, McCarthy MM, Sisk CL (2006) Dendritic pruning of the medial amygdala during pubertal development of the male Syrian hamster. J Neurobiol 66:578-590. CrossRef Medline 